archives-ouvertes

\title{
Finite element approximation of electromagnetic fields using nonfitting meshes for Geophysics
}

\author{
Théophile Chaumont-Frelet, Serge Nicaise, David Pardo
}

\section{To cite this version:}

Théophile Chaumont-Frelet, Serge Nicaise, David Pardo. Finite element approximation of electromagnetic fields using nonfitting meshes for Geophysics. SIAM Journal on Numerical Analysis, Society for Industrial and Applied Mathematics, In press, 56 (4), pp.2288-2321. <10.1137/16m1105566>. $<$ hal-01706452v2>

\author{
HAL Id: hal-01706452 \\ https://hal.inria.fr/hal-01706452v2
}

Submitted on 16 Dec 2018

HAL is a multi-disciplinary open access archive for the deposit and dissemination of scientific research documents, whether they are published or not. The documents may come from teaching and research institutions in France or abroad, or from public or private research centers.
L'archive ouverte pluridisciplinaire HAL, est destinée au dépôt et à la diffusion de documents scientifiques de niveau recherche, publiés ou non, émanant des établissements d'enseignement et de recherche français ou étrangers, des laboratoires publics ou privés. 


\title{
FINITE ELEMENT APPROXIMATION OF ELECTROMAGNETIC FIELDS USING NONFITTING MESHES FOR GEOPHYSICS*
}

\author{
THÉOPHILE CHAUMONT-FRELET ${ }^{\dagger}$, SERGE NICAISE $^{\ddagger}$, AND DAVID PARDO $^{\S}$
}

\begin{abstract}
We analyze the use of nonfitting meshes for simulating the propagation of electromagnetic waves inside the earth with applications to borehole logging. We avoid the use of parameter homogenization and employ standard edge finite element basis functions. For our geophysical applications, we consider a 3D Maxwell's system with piecewise constant conductivity and globally constant permittivity and permeability. The model is analyzed and discretized using both the Eand $\mathrm{H}$-formulations. Our main contribution is to develop a sharp error estimate for both the electric and magnetic fields. In the presence of singularities, our estimate shows that the magnetic field approximation is converging faster than the electric field approximation. As a result, we conclude that error estimates available in the literature are sharp with respect to the electric field error but provide pessimistic convergence rates for the magnetic field in our geophysical applications. Another surprising consequence of our analysis is that nonfitting meshes deliver the same convergence rate as fitting meshes to approximate the magnetic field. Our theoretical results are numerically illustrated via $2 \mathrm{D}$ experiments. For the analyzed cases, the accuracy loss due to the use of nonfitting meshes is limited, even for high conductivity contrasts.
\end{abstract}

Key words. Maxwell's equations, edge elements, nonfitting meshes, heterogeneous media, Aubin-Nitsche trick, geophysical applications

AMS subject classifications. Primary, 65N12, 65N15, 65N30; Secondary, 35Q61

DOI. $10.1137 / 16 \mathrm{M} 1105566$

Introduction. Electromagnetic (EM) methods are used in geophysics for reservoir characterization and, more generally, subsurface imaging. EM methods are sensitive to the high conductivity contrast that occurs between oil-saturated and water-saturated rocks. For this reason, they are routinely employed in geophysical surveys, such as well logging [18] (including laterolog [40], logging-while-drilling [39], through casing [41], and cross-well configurations [45]), controlled source EM [16], and magnetotellurics [44].

Forward modeling of the electromagnetic fields is a key aspect of the aforementioned applications. It is used either directly to compare a synthetic model to in-situ measurements, or as part of an inversion algorithm if the aim is to reconstruct a

*Received by the editors November 29, 2016; accepted for publication (in revised form) May 1, 2018; published electronically July 26, 2018.

http://www.siam.org/journals/sinum/56-4/M110556.html

Funding: The first and third authors were supported by the Projects of the Spanish Ministry of Economy and Competitiveness with reference MTM2016-76329-R and by the BCAM "Severo Ochoa" accreditation of excellence SEV-2013-0323. The third author was also supported by the European Union's Horizon 2020 research and innovation programme under the Marie Sklodowska-Curie grant agreement 644602, the Projects of the Spanish Ministry of Economy and Competitiveness with reference MTM2013-40824-P, the Basque Government through the BERC 2014-2017 program, the Consolidated Research Group grant IT649-13 on "Mathematical Modeling, Simulation, and Industrial Applications (M2SI)," and the ICERMAR Project KK-2015/0000097.

†BCAM - Basque Center for Applied Mathematics, 48009 Bilbao, Bizkaia, Spain (tchaumont@ bcamath.org).

${ }^{\ddagger}$ LAMAV and FR CNRS 2956, Université de Valenciennes et du Hainaut Cambrésis, Le Mont Houy, 59313 Valenciennes Cedex 9, France (snicaise@univ-valenciennes.fr).

$\S$ Department of Applied Mathematics, Statistics, and Operational Research, University of the Basque Country (UPV/EHU), 48940 Leioa, Bizkaia, Spain; BCAM - Basque Center for Applied Mathematics, 48009 Bilbao, Bizkaia, Spain; and Ikerbasque (Basque Foundation for Sciences), 48013 Bilbao, Bizkaia, Spain (dzubiaur@gmail.com). 
conductivity model from observed data [23]. In both cases, it is crucial to design a forward modeling method that is fast, accurate, and robust [3].

To describe the propagation of electromagnetic fields inside the earth, we consider 3D Maxwell's equations in a convex isotropic domain. The permittivity and permeability are assumed to be constant, and the conductivity is taken piecewise constant onto a polyhedral partition of the domain. Apart from the isotropy assumption, these hypotheses are rather general regarding geophysical applications.

Different discretization techniques are available to solve 3D Maxwell's equations [3]. Here, we focus on first order edge finite element (FE) discretizations (also known as Nédélec's elements [36]) and consider both electric and magnetic field formulations.

In FE methods, the mesh is typically aligned with the conductivity model. However, this mesh design constraint presents important limitations. On the other hand, nonfitting meshes permit us to simplify implementations and/or reduce computational costs. Figure 1 depicts a well logging application. As shown there, nonfitting meshes allow the user to design a single "sliding" mesh adapted to the tool configuration (sources, receivers, and skin-depth) and to use it for different tool positions. Other interesting applications include layers or shapes that are smaller than the mesh size, or taking into account inclined layers in a Cartesian grid mesh, as in [35]. More generally, in the context of inverse problems, nonfitting meshes enable the user to employ the same mesh for all iterations and to decouple the discretization of the forward problem with the conductivity model representation [23].

Here, we focus on the use of "nonfitting" meshes, where the conductivity may be discontinuous on the interior of an element. Standard Lagrange and Nédélec's elements are known to perform well only if the medium parameters are smooth inside each mesh cell. In the presence of nonfitting meshes, a usual technique is then to use homogenization or averaging formulae to upscale a varying parameter to one constant value inside each cell.

In the simplest case, the averaging consists of an explicit formula [30, 35, 24]. However, these averaging formulae are rigorously validated only under some specific assumptions, such as small-scale periodicity or one-dimensional variations. In order to overcome these difficulties, "numerical homogenization" techniques have been developed. The homogenized parameter can be obtained through the solution of a local problem inside each cell [19]. In general, numerical homogenization can be linked to multiscale methods, and the parameter upscaling algorithm can be viewed as a particular choice of basis functions satisfying a local boundary value problem $[26,42,4]$. For recent advances concerning multiscale methods and upscaling algorithms for Maxwell's equations, we refer the reader to [15, 25].

In the context of seismic wave propagation problems, similar averaging techniques are currently used $[5,10]$. Recently, a simpler technique, which avoids the use of averaging, homogenization, and multiscale basis functions, has been introduced by Chaumont-Frelet and collaborators for the propagation of acoustic waves inside the earth $[6,11]$. The main idea is to keep unmodified the basis functions and the earth model. Subcell variations of the model are then taken into account through accurate quadrature schemes. We will refer to this technique as the "exact quadrature technique" since the essence of the method is just to use standard FE shape functions and exactly (or at least, sufficiently accurately) integrate the corresponding variational formulation. In the context of seismic wave propagation, the exact quadrature technique is proven to be numerically efficient [13], and a convergence analysis shows that the method is well suited to approximate high-frequency waves, even if the solution can be rough inside the mesh cells [12]. The method is also compared to an averaging 


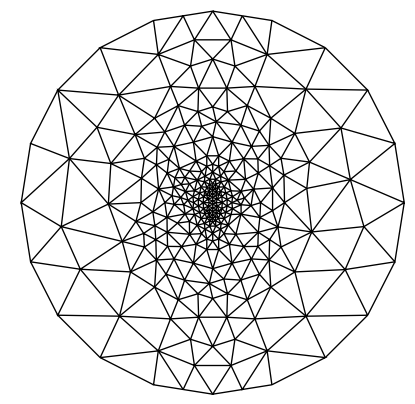

(a) Sliding mesh

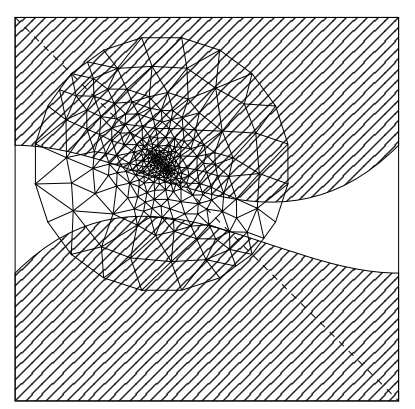

(d) Position 1

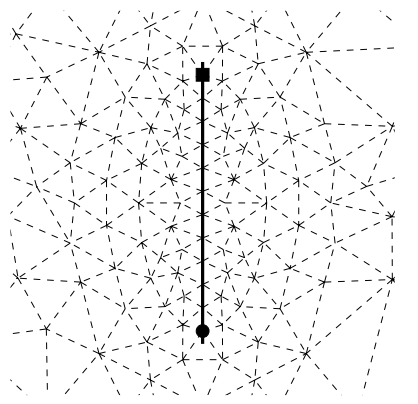

(b) Zoom around the logging tool

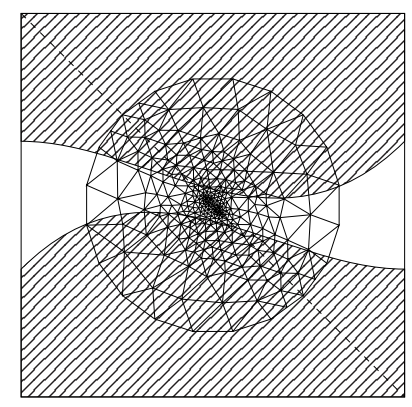

(e) Position 2

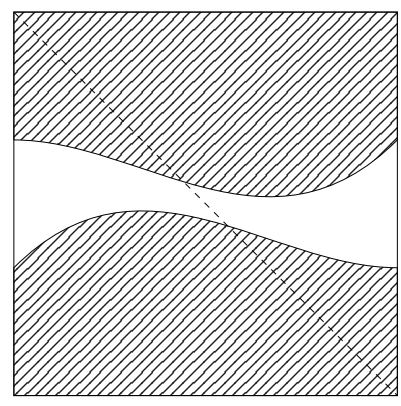

(c) Conductivity model and well trajectory

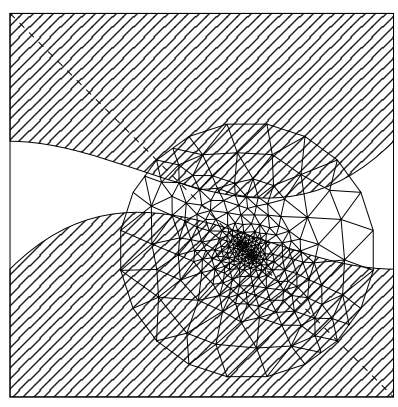

(f) Position 3

FIG. 1. Sliding mesh.

technique in [11], where it is shown that the exact integration technique outperforms averaging, especially when the characteristic size of the heterogeneities is not small enough compared to the solution wavelength.

The key point in the success of the exact quadrature technique for wave propagation is that most of the error is due to dispersion [12]. When considering Maxwell's system for geophysics, the situation is different, because the earth highly absorbs electromagnetic waves, so that dispersion errors are small. As a result, the main advantages and limitations of an exact quadrature technique for EM problems are unclear.

As presented in [31], a number of methods are currently proposed in the literature for solving smooth interface problems with nonfitting meshes. Specifically, two types of methods are described: standard curved FEs that approximate the interface "sufficiently well" and FE spaces with modified shape functions that can handle arbitrary meshes. Though the problem we consider has connections to those analyzed in [31], our work stands out as we consider arbitrary meshes (in the sense that we do not assume any approximation of the interfaces) with unmodified FE shape functions. Additionally, most of the methods mentioned in [31] target the Poisson equation and use Lagrange-type basis functions, while we focus on solving Maxwell's equations with Nédélec's basis functions.

In this work, we investigate the possible advantages of using the exact quadrature technique for borehole logging geophysical applications. To do so, we develop a rigorous convergence analysis of the aforementioned FE methods. Though our aim is to investigate nonfitting mesh discretizations, it turns out that our main result is 
also useful for fitting meshes. Since we focus on geophysical applications, the medium permeability is constant, and the magnetic field always exhibits $H^{1}$ regularity. On the other hand, because the conductivity is piecewise constant, the electric field only presents $H^{s}$ regularity, where $0<s \leq 1$ can be arbitrarily small, depending on the conductivity contrasts [17].

Recent $H$ (curl)-norm error estimates exist in the literature $[28,34]$. These results are optimal in the $H($ curl $)$-norm and predict an $\mathcal{O}\left(h^{s}\right)$ convergence rate in this configuration. The $H$ (curl)-norm can be viewed as a weighted sum of the $L^{2}$-norms of the electric and magnetic fields. Therefore, the aforementioned estimate predicts at least convergence rates of $\mathcal{O}\left(h^{s}\right)$ for both the electric and magnetic fields in the $L^{2}$-norm.

Here, we focus on proving optimal $L^{2}$ error estimates for both the electric and magnetic fields. We classically demonstrate that the electric field approximation converges as $\mathcal{O}\left(h^{s}\right)$. The novel result is that the magnetic field error decreases as $\mathcal{O}\left(h^{\min (1,2 s)}\right)$; its proof relies on a sort of Aubin-Nitsche trick.

An interesting consequence is that both fitting and nonfitting meshes deliver the same convergence rate for the magnetic field. In order to illustrate this feature, let us consider a layered medium without singularities. In this case [17], the electric field exhibits local $H^{1}$ regularity inside each layer but only $H^{(1-\delta) / 2}$ global regularity $(\delta>0$ being an arbitrarily small but strictly positive constant), since the normal component jumps across each interface. Because both fields are locally $H^{1}$, standard convergence arguments indicate linear convergence rates for both electromagnetic fields if a fitting mesh is used. If a nonfitting mesh is used, then the electric field only converges as $\mathcal{O}\left(h^{(1-\delta) / 2}\right)$. However, our new estimate shows that the magnetic field error decreases as $\mathcal{O}\left(h^{1-\delta}\right)$; that is, the convergence rate of the magnetic field approximation is almost linear, even for nonfitting meshes. This observation actually extends to general conductivity models with singularities. Hence, an important conclusion of our work is that fitting and nonfitting meshes virtually provide the same convergence rate for the magnetic field approximation.

Our convergence theory is validated and illustrated using 2D numerical experiments. We consider the $\mathrm{E}$ - and the $\mathrm{H}$-formulation in order to approximate transverse magnetic (TM) and transverse electric (TE) polarized problems, respectively. In these test cases, we compare the accuracy between fitting and nonfitting meshes as well as the impact of the conductivity contrast on the precision. These numerical tests show that our convergence analysis is sharp and that fitting and nonfitting meshes provide a comparable accuracy for the magnetic field approximation.

The proofs we provide are carried out for the 3D Maxwell's system, and the techniques we use are typical of FE analysis. The main conclusion stating that increased accuracy can be expected for the magnetic field shall be useful in analyzing other discretization techniques, such as finite differences [3], and might give additional insight when deriving homogenization formulae. Also, we expect our conclusions to hold true for simplified EM models such as the 2.5D Maxwell's system [2]. In addition, though we essentially focus on borehole logging, we believe that our main conclusions will also apply to other EM geophysical applications, such as controlled source EM and magnetotellurics.

Issues related to nonfitting meshes arise in a variety of methods, such as discontinuous Galerkin [37] or nonconforming [9] discretizations. The aim of our work is thus not to discuss the efficiency of a particular discretization strategy but to study the usefulness of nonfitting meshes. Hence, though our results are rigorously established only for FE discretizations, we believe they are insightful for a range of mesh-based discretization methods. 
The paper is organized as follows. In section 1, we introduce Maxwell's equations and analyze the regularity of their solutions. We introduce the FE spaces and carry out the error analysis in section 2. Finally, numerical results are presented in section 3, confirming our predicted convergence rates and the efficiency of the proposed numerical methods.

\section{Maxwell's equations.}

1.1. Notation. Hereafter, $\Omega \subset \mathbb{R}^{3}$ is a bounded convex polyhedral open set. The symbol $\partial \Omega$ is used to denote the boundary of $\Omega$, and $\mathbf{n}$ is the unit vector normal to $\partial \Omega$ and pointing outside $\Omega$.

We assume that $\Omega$ is covered by a partition $\mathscr{P}=\left\{\Omega_{k}\right\}_{k=1}^{S}$ of polyhedron $\Omega_{k}$. Then, the conductivity model $\sigma: \Omega \rightarrow \mathbb{R}_{+}^{*}$ takes one constant positive value $\sigma_{k}$ on each $\Omega_{k}$. We define $\sigma_{\star}=\min _{k} \sigma_{k}$ and $\sigma^{\star}=\max _{k} \sigma_{k}$. By assumption, we have $0<\sigma_{\star} \leq \sigma^{\star}<+\infty$.

We denote by $\mathcal{F}$ the set of faces related to the partition $\mathscr{P}$. For each face $F \in \mathcal{F}$ we define an associated normal vector $\mathbf{n}_{F}$ such that $\mathbf{n}_{F}=\mathbf{n}$ if $F \subset \partial \Omega$. Also, for each $k \in\{1, \ldots, S\}$, we denote by $\mathbf{n}_{k}$ the unit vector normal to $\partial \Omega_{k}$ and pointing outside $\Omega_{k}$. The jump of $\sigma$ through a face $F \in \mathcal{F}$ is defined as

$$
[[\sigma]]_{F}= \begin{cases}\left(\sigma_{k} \mathbf{n}_{k}+\sigma_{j} \mathbf{n}_{j}\right) \cdot \mathbf{n}_{F} & \text { if } F=\partial \Omega_{k} \cap \partial \Omega_{j} \\ \sigma_{k} & \text { if } F=\partial \Omega_{k} \cap \partial \Omega\end{cases}
$$

Throughout the rest of this paper, symbol $h$ will be reserved to denote the FE mesh size. Also, $\beta \in(0,1)$ will be a fixed constant representing the geometric regularity of the FE meshes (see (20) in section 2.1), and $\delta>0$ will be a strictly positive, arbitrarily small constant. If $A, B$ are positive real numbers, we shall write $A \lesssim B$ if there exists a constant $C(\Omega, \mathscr{P}, \sigma, \beta, \delta)$ depending on $\Omega, \mathscr{P}, \sigma, \beta$, and $\delta$ but independent of $h$, such that $A \leq C(\Omega, \mathscr{P}, \sigma, \beta, \delta) B$.

For an arbitrary open set $\mathcal{U} \subset \mathbb{R}^{d}$ with $d=2$ or $3, L^{2}(\mathcal{U})$ is the space of square integrable, complex-valued functions. For $s \in \mathbb{R}$, we classically denote by $H^{s}(\mathcal{U})$ the Sobolev space of complex-valued function defined in $\mathcal{U}$. We also introduce the spaces of vector functions $\mathbf{H}^{s}(\mathcal{U})=\left(H^{s}(\mathcal{U})\right)^{3}$ and $\mathbf{L}^{2}(\mathcal{U})=\left(L^{2}(\mathcal{U})\right)^{3}$. We use the notation $\|\cdot\|_{s, \mathcal{U}}$ and $|\cdot|_{s, \mathcal{U}}$ for the usual norm and seminorm of $H^{s}(\mathcal{U})$ and $\mathbf{H}^{s}(\mathcal{U})$. The notation $(.,.) \mathcal{U}$ is used for the $L^{2}(\mathcal{U})$ and $\mathbf{L}^{2}(\mathcal{U})$ inner-products. We drop the domain subscript if $\mathcal{U}=\Omega$. We refer the reader to $[1,32]$ for a discussion of these spaces.

If $\mathbf{u} \in \mathbf{L}^{2}(\mathcal{U}), \nabla \cdot \mathbf{u}$ and $\nabla \times \mathbf{u}$ denote its divergence and rotational in the sense of distributions, respectively. $\mathbf{H}(\operatorname{div}, \mathcal{U})$ and $\mathbf{H}(\operatorname{curl}, \mathcal{U})$ are the subspaces of $\mathbf{L}^{2}(\mathcal{U})$ having square integrable divergence and rotational, respectively [22]. These spaces are embedded with the norms

$$
\|\mathbf{u}\|_{\text {div }, \mathcal{U}}^{2}=|\mathbf{u}|_{0, \mathcal{U}}^{2}+|\nabla \cdot \mathbf{u}|_{0, \mathcal{U}}^{2} \quad \forall \mathbf{u} \in \mathbf{H}(\operatorname{div}, \mathcal{U})
$$

and

$$
\|\mathbf{u}\|_{c u r l, \mathcal{U}}^{2}=|\mathbf{u}|_{0, \mathcal{U}}^{2}+|\nabla \times \mathbf{u}|_{0, \mathcal{U}}^{2} \quad \forall \mathbf{u} \in \mathbf{H}(\operatorname{curl}, \mathcal{U})
$$

We denote by $\mathcal{D}(\mathcal{U})$ the space of $C^{\infty}$ functions having compact support in $\mathcal{U}$. If $\mathcal{H}(\mathcal{U})$ is any of the aforementioned scalar (resp., vectorial) Sobolev spaces, then $\mathcal{H}_{0}(\mathcal{U})$ denotes the closure of $\mathcal{D}(\mathcal{U})$ (resp., $\left.(\mathcal{D}(\mathcal{U}))^{3}\right)$ in $\mathcal{H}(\mathcal{U})$.

Finally, for $s \in \mathbb{R}_{+}$, we shall also use "broken" Sobolev spaces of piecewise smooth 
functions onto the partition $\mathscr{P}$. These spaces are defined as

$$
P H^{s}(\Omega, \mathscr{P})=\left\{u \in L^{2}(\Omega) \mid u \in H^{s}\left(\Omega_{k}\right) \forall \Omega_{k} \in \mathscr{P}\right\}
$$

and $\mathbf{P} \mathbf{H}^{s}(\Omega, \mathscr{P})=\left(P H^{s}(\Omega)\right)^{3}$. If $s<1 / 2$, then $P H^{s}(\Omega, \mathscr{P})=H^{s}(\Omega)$. For $s \geq 1 / 2$, if $u \in P H^{s}(\Omega, \mathscr{P})$, then $u \in H^{1 / 2-\delta}(\Omega)$. Analogous properties hold for the vectorial broken spaces [32].

1.2. Model. For a given electric source $\mathbf{J}$ and magnetic source $\mathbf{M}$, Maxwell's equations governing the electromagnetic fields for a nonzero frequency $\omega$ read

$$
\left\{\begin{aligned}
i \omega \mu_{0} \mathbf{H}-\nabla \times \mathbf{E} & =\mathbf{M} \\
(\sigma+i \omega \varepsilon) \mathbf{E}+\nabla \times \mathbf{H} & =\mathbf{J}
\end{aligned}\right.
$$

in $\Omega$. In addition, the following conditions are imposed on the boundary $\partial \Omega$ :

$$
\mathbf{E} \times \mathbf{n}=\mathbf{0}, \quad \mathbf{H} \cdot \mathbf{n}=0 .
$$

In the above model, we assume that the permeability $\mu$ is constant and set to the vacuum value $\mu_{0}=4 \pi \times 10^{-7} N A^{-2}$. Also, following [44], we assume that $\sigma+i \omega \varepsilon \simeq \sigma$, so that $\varepsilon$ is set to zero. Actually, the only hypothesis that is mandatory on the parameters is that $\mu$ is constant. But to simplify notation in the proofs, we consider $\varepsilon=0$.

We assume that all parameters are scalar valued, which corresponds to an isotropic propagation domain. This hypothesis is actually restrictive because most rocks are anisotropic. However, the isotropy hypothesis is mostly made to simplify the analysis (in particular, we can apply the results presented in [17]). Indeed, the extension of the proposed FE methods to anisotropic propagation media is straightforward.

As detailed before, the domain $\Omega$ is a convex polyhedron. In most geophysical applications, this hypothesis is rather realistic, because the boundary of the domain is not physical but artificially designed to bound the computational domain. The size of the domain is related to the skin-depth relation [44], and the shape of the domain is generally a cube or a sphere-like polyhedron. For a nonconvex domain, re-entrant corners might generate singularities that are not considered in the present analysis. We refer the reader to $[17,7,28,38]$.

The assumptions we make about the medium of propagation are justified by the type of applications we consider. Let us further describe each of them:

1. The assumption that $\varepsilon=0$ (which is common in the geophysical applications we consider) is only made to simplify notation in the paper, and our results hold unchanged as long as $\sigma>0$. On the other hand, the case corresponding to purely dispersive medium with $\varepsilon>0$ and $\sigma=0$ is much more difficult to analyze. In the following, we briefly mention some of the difficulties that arise in this last setting, and we refer the reader to [33, 34] for additional details:

- Unless boundary conditions are modified, there exist resonance frequencies for which the problem is not well-posed.

- When the problem is well-posed, apart from frequencies below the first resonance frequency, the sesquilinear form associated with the boundary value problem is not coercive, which greatly complexifies the discretization.

- The loss of coercivity manifests itself by the so-called "pollution effect": discrete stability is lost, unless the mesh is sufficiently refined [27]. As a result, quasi-optimality of the FE solution is lost, apart from an "asymptotic range" (corresponding to sufficiently refined meshes). 
In this work, we focus on the easiest case where $\sigma>0$. As a result, the problem is well-posed and the sesquilinear forms are coercive at all frequencies. This means that the pollution effect is avoided, and that the FE is quasi-optimal for arbitrary meshes at all frequencies (we note, however, that the stability constant depends on the ratio $\max \varepsilon / \min \sigma)$. Though we believe our main results could help characterize the "asymptotic range" and derive asymptotic error estimates in the case where $\sigma=0$, we do not address this case here, as it brings multiple additional difficulties.

2. The assumption that $\mu$ is constant (or at least smooth) is fundamental, as it allows for the magnetic field to be regular. A symmetric situation exists if we assume that $\mu$ is variable and $\varepsilon$ and $\sigma$ are constant, for which the convergence rate of the electric field is improved. Here, we focus on the case where $\mu=\mu_{0}$, as it is a typical assumption for the applications we consider.

3. The case where $\Omega$ is not convex is unrelated to the use of nonfitting meshes, in the sense that all the meshes we consider perfectly match the boundary. Then, for both fitting and nonfitting meshes, singularities can occur close to the boundary of $\Omega$, and our main results essentially hold unchanged, as long as the meshes are geometrically refined close to the singular edges and vertices of the boundary (see $[17,38]$ ).

4. Dirichlet boundary conditions are satisfactory to bound the computational domain for the borehole applications we consider, since the EM fields decay fast away from the emitter. On the other hand, some applications require the Silver-Müller radiation condition to be approximated with elaborated boundary conditions (see [34], for instance), such as absorbing boundary conditions $(\mathrm{ABC})$ or perfectly matched layers (PML). We do not address these boundary conditions as they make the analysis more complex:

- For ABC, dedicated functional spaces with well-defined traces are required. These functional spaces are more complicated to handle than the standard $\mathbf{H}(\mathrm{curl}, \Omega)$ space we employ.

- For PML, the coefficients $\varepsilon$ and $\mu$ are artificially modified. Then, we have to consider a varying $\mu$, which we avoid here for the sake of simplicity.

Nevertheless, the authors do not foresee fundamental reasons that would prevent the improved convergence rate of the magnetic field to occur with these boundary conditions.

Assuming that $\mathbf{J}, \mathbf{M} \in \mathbf{L}^{2}(\Omega)$, we can prove existence and uniqueness of weak solutions $\mathbf{E}, \mathbf{H} \in \mathbf{H}(\mathrm{curl}, \Omega)$ to (1)-(2) using the variational framework (see, for instance, [34]). We recast the first order system (1)-(2) into a second order system, having only one unknown. As the original system features two unknowns, two choices are possible.

The electric field formulation is obtained by eliminating $\mathbf{H}$ from (1)-(2). Then, the equation for the electric field reads

$$
\left\{\begin{aligned}
i \omega \mu_{0} \sigma \mathbf{E}+\nabla \times \nabla \times \mathbf{E} & =i \omega \mu_{0} \mathbf{J}-\nabla \times \mathbf{M} & & \text { in } \Omega, \\
\mathbf{E} \times \mathbf{n} & =\mathbf{0} & & \text { on } \partial \Omega .
\end{aligned}\right.
$$

In the sense of distributions, boundary value problem (3) is equivalent to finding $\mathbf{E} \in \mathbf{H}_{0}(\mathrm{curl}, \Omega)$ such that

(4) $i \omega \mu_{0}(\sigma \mathbf{E}, \mathbf{v})+(\nabla \times \mathbf{E}, \nabla \times \mathbf{v})=i \omega \mu_{0}(\mathbf{J}, \mathbf{v})-(\mathbf{M}, \nabla \times \mathbf{v}) \quad \forall \mathbf{v} \in \mathbf{H}_{0}(\operatorname{curl}, \Omega)$

Alternatively, for the magnetic field formulation, we look for $\mathbf{H} \in \mathbf{H}(\operatorname{curl}, \Omega)$ 
satisfying

(5)

$$
i \omega \mu_{0}(\mathbf{H}, \mathbf{v})+\left(\sigma^{-1} \nabla \times \mathbf{H}, \nabla \times \mathbf{v}\right)=(\mathbf{M}, \mathbf{v})+\left(\sigma^{-1} \mathbf{J}, \nabla \times \mathbf{v}\right) \quad \forall \mathbf{v} \in \mathbf{H}(\operatorname{curl}, \Omega) .
$$

In order to shorten notation, we introduce the sesquilinear forms

$$
b_{\mathbf{E}}(\mathbf{u}, \mathbf{v})=i \omega \mu_{0}(\sigma \mathbf{u}, \mathbf{v})+(\nabla \times \mathbf{u}, \nabla \times \mathbf{v}) \quad \forall \mathbf{u}, \mathbf{v} \in \mathbf{H}_{0}(\operatorname{curl}, \Omega)
$$

and

$$
b_{\mathbf{H}}(\mathbf{u}, \mathbf{v})=i \omega \mu_{0}(\mathbf{u}, \mathbf{v})+\left(\sigma^{-1} \nabla \times \mathbf{u}, \nabla \times \mathbf{v}\right) \quad \forall \mathbf{u}, \mathbf{v} \in \mathbf{H}(\operatorname{curl}, \Omega),
$$

associated with (4) and (5).

We start by introducing some technical results that will be of use for the proofs of regularity of solutions.

1.3. Technical preliminary results. When analyzing the solutions of Maxwell's system (1), the variational theory easily shows that $\mathbf{E}, \mathbf{H} \in \mathbf{H}(\mathrm{curl}, \Omega)$ assuming that $\mathbf{J}, \mathbf{M} \in \mathbf{L}^{2}(\Omega)$. A more subtle issue is to find optimal values $0<s, s^{\prime} \leq 1$ such that $\mathbf{E} \in \mathbf{P} \mathbf{H}^{s}(\Omega, \mathscr{P})$ and $\mathbf{H} \in \mathbf{P} \mathbf{H}^{s^{\prime}}(\Omega, \mathscr{P})$.

The exponents $s, s^{\prime}$ depend on the smoothness of the domain and the coefficients $\varepsilon, \mu$, and $\sigma$. In our settings, because $\mu$ is constant and $\Omega$ is convex, we can easily show that $\mathbf{H} \in \mathbf{H}^{1}(\Omega)$ under the assumption that $\mathbf{M} \in \mathbf{H}(\operatorname{div}, \Omega)$, so that $s^{\prime}=1$. However, the regularity of $\mathbf{E}$ will be harder to analyze, and we shall require technical results from [17, 7]. Here, the singularities of $\mathbf{E}$ are due to the conductivity parameter $\sigma$. Hence, we will denote by $\tau(\sigma)$ the highest value so that $\mathbf{E} \in \mathbf{P H}^{s}(\Omega, \mathscr{P})$ for all $0<s<\tau(\sigma)$.

The convergence rate of $\mathrm{FE}$ discretizations mostly depend on the elementwise regularity of $\mathbf{E}$. The elementwise regularity corresponds to $\tau(\sigma)$ for fitting meshes. However, for nonfitting meshes, this elementwise regularity is given by $\min (\tau(\sigma), 1 / 2)$. This is the reason why fitting and nonfitting meshes may exhibit different convergence rates.

The characterization of $\tau(\sigma)$ is rather involved $[17,7]$. As we depict hereafter, it is linked to the Poisson equation with coefficient $\sigma$.

The regularity of the electric field is analyzed through a Helmholtz-Hodge decomposition $\mathbf{E}=\boldsymbol{\psi}+\nabla p$, where $\boldsymbol{\psi} \in \mathbf{H}_{0}(\mathrm{curl}, \Omega)$ is divergence free and $p \in H_{0}^{1}(\Omega)$. In our settings, it turns out that $\boldsymbol{\psi} \in \mathbf{H}^{1}(\Omega)$, so that the singularities of $\mathbf{E}$ are contained in $p$. Furthermore (see [17]), $p \in H_{0}^{1}(\Omega)$ can be characterized as the solution of a "Poisson type" variational problem,

$$
(\sigma \nabla p, \nabla q)=(f, q)+\sum_{F \in \mathcal{F}}\left(g_{F}, q\right)_{F} \quad \forall q \in H_{0}^{1}(\Omega),
$$

where $f \in L^{2}(\Omega)$ and $g_{F} \in H^{1 / 2}(F)$ for all $F \in \mathcal{F}$. As depicted in the proof of Theorem 2, $f$ and $g$, respectively, depend on $\mathbf{J}$ and $\boldsymbol{\psi}$, and one can show (see [17]) that

$$
p \in P H^{1+s}(\Omega, \mathscr{P})
$$

for some $0<s \leq 1$, with continuous dependence. Then, $\tau(\sigma)$ is the highest value such that (7) holds for all $0<s<\tau(\sigma)$.

Boundary value problem (6) is rather unusual, because its right-hand side involves surface sources on the interfaces of partition $\mathscr{P}$, and as a result it does not belong to 
$L^{2}(\Omega)$. But Theorem 4.1 of [17] shows that the regularity of the solution $p$ of (6) is the same as the regularity of $u \in H_{0}^{1}(\Omega)$ solution to

$$
(\sigma \nabla u, \nabla v)=(f, v) \quad \forall v \in H_{0}^{1}(\Omega),
$$

with $f \in L^{2}(\Omega)$. Hence, we can think of the electric field regularity as that of the gradient of a solution to the standard Poisson equation (8). Note that $\tau(\sigma)$ also corresponds to the convergence rates of a Lagrange $\mathrm{FE}$ method in the $H^{1}$-norm for the case of fitting meshes [8, Thm. 9.25].

An important particular case occurs when the conductivity model is made of horizontal layers in a cubic domain. Indeed, in this configuration, we have $\tau(\sigma)=2$ (for smooth enough right-hand side), and in particular $\mathbf{E} \in \mathbf{P H}^{1}(\Omega, \mathscr{P})$ (see [17]). For other configurations, some lower bounds for $\tau(\sigma)$ based on the values of $\sigma$ are presented in the appendix of [17].

We also refer the reader to [7] for regularity results in more general domains and medium parameters.

1.4. Electric field formulation. We start with an existence and uniqueness result for (4). The analysis is greatly simplified because we assume that the conductivity is strictly positive. As a result, the proof of the following theorem relies simply on the Lax-Milgram lemma and avoids the use of Fredholm alternative and compact embeddings (see Chapter 4 of [34]).

Theorem 1. There exists a unique solution $\mathbf{E} \in \mathbf{H}_{0}($ curl,$\Omega)$ to (4). Furthermore, it holds that

$$
\|\mathbf{E}\|_{\text {curl }} \lesssim|\mathbf{J}|_{0}+|\mathbf{M}|_{0} .
$$

Proof. If $\mathbf{v} \in \mathbf{H}_{0}(\operatorname{curl}, \Omega)$, we have

$$
b_{\mathbf{E}}(\mathbf{v}, \mathbf{v})=i \omega \mu_{0}(\sigma \mathbf{v}, \mathbf{v})+(\nabla \times \mathbf{v}, \nabla \times \mathbf{v})=i \omega \mu_{0}\left|\sigma^{1 / 2} \mathbf{v}\right|_{0}^{2}+|\nabla \times \mathbf{v}|_{0}^{2},
$$

and it follows that

$$
\left|b_{\mathbf{E}}(\mathbf{v}, \mathbf{v})\right| \gtrsim\|\mathbf{v}\|_{\text {curl }}^{2} .
$$

Inequality (10) implies that the sesquilinear form $b_{\mathbf{E}}$ is coercive. It is also easily seen that $b_{\mathbf{E}}$ is continuous. Finally, because $\mathbf{J}, \mathbf{M} \in \mathbf{L}^{2}(\Omega)$, the functional

$$
\mathbf{H}_{0}(\operatorname{curl}, \Omega) \ni \mathbf{v} \rightarrow i \omega \mu_{0}(\mathbf{J}, \mathbf{v})-(\mathbf{M}, \nabla \times \mathbf{v}) \in \mathbb{C}
$$

is an element of $\left(\mathbf{H}_{0}(\mathrm{curl}, \Omega)\right)^{\prime}$. As a result, we can apply the Lax-Milgram lemma (see, for instance, Chapter 2 of [34]), and the existence and uniqueness of $\mathbf{E}$ follows.

To prove (9), we select $\mathbf{v}=\mathbf{E}$ in (4) and (10). Then, we have

$$
\|\mathbf{E}\|_{\text {curl }}^{2} \lesssim\left|b_{\mathbf{E}}(\mathbf{E}, \mathbf{E})\right|=\left|i \omega \mu_{0}(\mathbf{J}, \mathbf{E})-(\mathbf{M}, \nabla \times \mathbf{E})\right| \lesssim\left(|\mathbf{J}|_{0}+|\mathbf{M}|_{0}\right)\|\mathbf{E}\|_{\text {curl }} .
$$

Now, we show that if additional regularity is assumed on $\mathbf{J}$ and/or $\mathbf{M}$, additional regularity can be expected for the electric field. The proof involves the exponent $\tau(\sigma)$ associated with the Poisson equation that is introduced in subsection 1.3.

Theorem 2. If $\mathbf{J} \in \mathbf{H}($ div,$\Omega)$, then $\mathbf{E} \in \mathbf{P} \mathbf{H}^{s}(\Omega, \mathscr{P})$, and it holds that

$$
\sum_{k=1}^{S}|\mathbf{E}|_{s, \Omega_{k}} \lesssim\|\mathbf{J}\|_{d i v}+|\mathbf{M}|_{0}
$$


for all $0<s<\tau(\sigma)$. Also $\mathbf{E} \in \mathbf{H}^{t}(\Omega)$, and we have

$$
|\mathbf{E}|_{t} \lesssim\|\mathbf{J}\|_{\text {div }}+|\mathbf{M}|_{0}
$$

for all $0<t<\tilde{\tau}(\sigma)=\min (\tau(\sigma), 1 / 2)$.

If $\mathbf{M} \in \mathbf{H}($ curl,,$\Omega)$, then $\nabla \times \mathbf{E} \in \mathbf{H}^{1}(\Omega)$, and it holds that

$$
|\nabla \times \mathbf{E}|_{1} \lesssim|\mathbf{J}|_{0}+\|\mathbf{M}\|_{\text {curl }}
$$

Proof. As depicted in section 1.3, we have $\mathbf{E}=\nabla p+\boldsymbol{\psi}$ with $\boldsymbol{\psi} \in \mathbf{H}_{0}(\mathrm{curl}, \Omega)$, $\nabla \cdot \boldsymbol{\psi}=0$, and $p \in H_{0}^{1}(\Omega)$. We select a test function $\mathbf{v}=\nabla q$ in (4) and see that $p$ is the solution to

$$
(\sigma \nabla p, \nabla q)=(\mathbf{J}-\sigma \boldsymbol{\psi}, \nabla q) \quad \forall q \in H_{0}^{1}(\Omega) .
$$

Theorem I.3.7 of [22] ensures that $\boldsymbol{\psi} \in \mathbf{H}^{1}(\Omega)$ and $\|\boldsymbol{\psi}\|_{1} \lesssim|\nabla \times \mathbf{E}|_{0}$. Hence, it remains to study the regularity of $p$. We will show that $p \in P H^{1+s}(\Omega, \mathscr{P})$ and

$$
\sum_{k=1}^{S}|p|_{1+s, \Omega_{k}} \lesssim|\nabla \cdot \mathbf{J}|_{0}+|\nabla \times \mathbf{E}|_{0}
$$

for all $0<s<\tau(\sigma)$, which proves (11), since $|\nabla \times \mathbf{E}|_{0}$ has already been bounded in Theorem 1 .

Since $\boldsymbol{\psi} \in \mathbf{H}^{1}(\Omega)$, by applying Green's formula in each $\Omega_{k}$, we have

$$
\begin{aligned}
(\sigma \boldsymbol{\psi}, \nabla q) & =\sum_{k=1}^{S} \sigma_{k} \int_{\Omega_{k}} \boldsymbol{\psi} \cdot \nabla q \\
& =\sum_{k=1}^{S} \sigma_{k}\left\{\int_{\partial \Omega_{k}} \boldsymbol{\psi} \cdot \mathbf{n}_{K} q-\int_{\Omega_{k}} \nabla \cdot \boldsymbol{\psi} q\right\}
\end{aligned}
$$

Because $\nabla \cdot \boldsymbol{\psi}=0$, the second term vanishes. Furthermore, we can rearrange the first term so that

$$
(\sigma \boldsymbol{\psi}, \nabla q)=\sum_{F \in \mathcal{F}}[[\sigma]]_{F} \int_{F} \boldsymbol{\psi} \cdot \mathbf{n}_{F} q
$$

We thus find that $p \in H_{0}^{1}(\Omega)$ is a solution to (6) with $f=-\nabla \cdot \mathbf{J} \in L^{2}(\Omega)$ and $g_{F}=-[[\sigma]]_{F} \boldsymbol{\psi} \cdot \mathbf{n}_{F} \in H^{1 / 2}(F)$ for all $F \in \mathcal{F}_{\text {int }}$.

According to [17], it follows that for $0<s<\tau(\sigma), p \in P H^{1+s}(\Omega, \mathscr{P})$, with

$$
\sum_{k=1}^{S}|p|_{1+s, \Omega_{k}} \lesssim|\nabla \cdot \mathbf{J}|_{0}+\sum_{F \in \mathcal{F}_{\text {int }}}[[\sigma]]_{F}\left\|\boldsymbol{\psi} \cdot \mathbf{n}_{F}\right\|_{1 / 2, F}
$$

Therefore, by a trace theorem, we obtain

$$
\sum_{k=1}^{S}|p|_{1+s, \Omega_{k}} \lesssim|\nabla \cdot \mathbf{J}|_{0}+\|\boldsymbol{\psi}\|_{1},
$$

and we recover (11) by the previous estimate on $\|\boldsymbol{\psi}\|_{1}$. 
Recalling that $\tilde{\tau}(\sigma)=\min (\tau(\sigma), 1 / 2)$, we have $\tilde{\tau}(\sigma) \leq \tau(\sigma)$, so that $\mathbf{E} \in \mathbf{P} \mathbf{H}^{t}(\Omega, \mathscr{P})$ for all $0<t<\tilde{\tau}(\sigma)$. As $\tilde{\tau}(\sigma)<1 / 2$, we have $\mathbf{P H}^{t}(\Omega, \mathscr{P})=\mathbf{H}^{t}(\Omega)$, and (12) follows.

We now prove (13). In order to simplify notation, we write $\phi=\nabla \times \mathbf{E} \in \mathbf{L}^{2}(\Omega)$. Assuming that $\mathbf{M} \in \mathbf{H}(\mathrm{curl}, \Omega)$, we can write from (4) that

$$
(\phi, \nabla \times \mathbf{v})=\left(-i \omega \mu_{0} \sigma \mathbf{E}+i \omega \mu_{0} \mathbf{J}-\nabla \times \mathbf{M}, \mathbf{v}\right) \quad \forall \mathbf{v} \in \mathbf{H}_{0}(\operatorname{curl}, \Omega) .
$$

As a result, we have $\nabla \times \phi \in \mathbf{L}^{2}(\Omega)$ and

$$
|\nabla \times \phi|_{0} \leq \omega \mu_{0} \sigma^{\star}|\mathbf{E}|_{0}+\omega \mu_{0}|\mathbf{J}|_{0}+|\nabla \times \mathbf{M}|_{0} .
$$

From (9), it follows that

$$
|\nabla \times \phi|_{0} \lesssim|\mathbf{J}|_{0}+|\mathbf{M}|_{0}+|\nabla \times \mathbf{M}|_{0}
$$

As $\phi=\nabla \times \mathbf{E}$, we have $\nabla \cdot \phi=0$ on $\Omega$ and $\phi \cdot \mathbf{n}=0$ on $\partial \Omega$. Hence, applying Theorem I.3.9 from [22], we obtain (13).

1.5. Magnetic field formulation. In the previous subsection, we have reformulated the first order system (1)-(2) into second order boundary value problem (4) in terms of the electric field. In this subsection, we study the analogous formulation (5) in terms of the magnetic field.

In Theorem 3, we state an existence and uniqueness result for the magnetic field. The proof is similar to the electric field formulation and is not reproduced here.

TheOrem 3. There exists a unique solution $\mathbf{H} \in \mathbf{H}($ curl, $\Omega)$ to (5). Furthermore, it holds that

$$
\|\mathbf{H}\|_{\text {curl }} \lesssim|\mathbf{J}|_{0}+|\mathbf{M}|_{0} .
$$

Following the analysis of the electric field formulation, we show that if additional regularity is assumed on $\mathbf{J}$ and/or $\mathbf{M}$, the regularity of $\mathbf{H}$ is improved. Here again, the exponent $\tau(\sigma)$ defined earlier plays a crucial role.

Theorem 4. If $\mathbf{M} \in \mathbf{H}_{0}($ div,$\Omega)$, then $\mathbf{H} \in \mathbf{H}^{1}(\Omega)$, and it holds that

$$
|\mathbf{H}|_{1} \lesssim|\mathbf{J}|_{0}+\|\mathbf{M}\|_{\text {div }}
$$

If $\sigma^{-1} \mathbf{J} \in \mathbf{H}_{0}(\operatorname{curl}, \Omega)$, then $\nabla \times \mathbf{H} \in \mathbf{P} \mathbf{H}^{s}(\Omega, \mathscr{P})$, and it holds that

$$
\sum_{k=1}^{S}|\nabla \times \mathbf{H}|_{s, \Omega_{k}} \lesssim\left\|\sigma^{-1} \mathbf{J}\right\|_{\text {curl }}+|\mathbf{M}|_{0}
$$

for all $0<s<\tau(\sigma)$. Consequently $\nabla \times \mathbf{H} \in \mathbf{H}^{t}(\Omega)$ and

$$
|\nabla \times \mathbf{H}|_{t} \lesssim\left\|\sigma^{-1} \mathbf{J}\right\|_{\text {curl }}+|\mathbf{M}|_{0}
$$

for all $0<t<\tilde{\tau}(\sigma)=\min (\tau(\sigma), 1 / 2)$.

Proof. We first show (15). Taking $\mathbf{v}=\nabla p$ with $p \in H^{1}(\Omega)$ in (5), we have that

$$
i \omega \mu_{0}(\mathbf{H}, \nabla p)=(\mathbf{M}, \nabla p) .
$$

Since $\mathbf{M} \in \mathbf{H}_{0}(\operatorname{div}, \Omega), \mathbf{H} \in \mathbf{H}_{0}(\operatorname{div}, \Omega)$ and $\nabla \cdot \mathbf{H}=\nabla \cdot \mathbf{M} \in L^{2}(\Omega)$. Recalling (14), we also have that $\nabla \times \mathbf{H} \in \mathbf{L}^{2}(\Omega)$, and (15) follows from Theorem I.3.9 of [22]. 
In order to prove (16), we define $\mathbf{R}=\sigma^{-1} \nabla \times \mathbf{H}$. Since $\mathbf{H} \in \mathbf{H}($ curl,$\Omega)$ and $\sigma^{-1} \leq$ $\sigma_{\star}^{-1}<+\infty$, we have $\mathbf{R} \in \mathbf{L}^{2}(\Omega)$. Furthermore, because $\nabla \times\left(\sigma^{-1} \mathbf{J}\right) \in \mathbf{H}_{0}(\mathrm{curl}, \Omega)$, a simple manipulation of (5) shows that

$$
(\mathbf{R}, \nabla \times \mathbf{v})=\left(\mathbf{M}+\nabla \times\left(\sigma^{-1} \mathbf{J}\right)-i \omega \mu_{0} \mathbf{H}, \mathbf{v}\right) \quad \forall \mathbf{v} \in \mathbf{H}(\operatorname{curl}, \Omega) .
$$

It follows that $\mathbf{R} \in \mathbf{H}_{0}(\mathrm{curl}, \Omega)$. Let us introduce the Hodge-Helmholtz decomposition $\mathbf{R}=\boldsymbol{\psi}+\nabla p$, where $\boldsymbol{\psi} \in \mathbf{H}_{0}(\mathrm{curl}, \Omega)$ satisfies $\nabla \cdot \boldsymbol{\psi}=0$ and $p \in H_{0}^{1}(\Omega)$.

Since $\Omega$ is convex, Theorem I.3.7 of [22] ensures that $\boldsymbol{\psi} \in \mathbf{H}^{1}(\Omega)$ and $\|\boldsymbol{\psi}\|_{1} \lesssim$ $|\nabla \times \mathbf{R}|_{0}$. Recalling (18) and (14), it follows that

$$
\|\boldsymbol{\psi}\|_{1} \lesssim\left\|\sigma^{-1} \mathbf{J}\right\|_{\text {curl }}+|\mathbf{M}|_{0}
$$

It remains to analyze $p$, which contains the singularities. To do so, we observe that since $\sigma \mathbf{R}=\nabla \times \mathbf{H}$, we have $(\sigma \mathbf{R}, \nabla q)=0$ for all $q \in H_{0}^{1}(\Omega)$. As a result, we see that $p$ is the unique solution in $H_{0}^{1}(\Omega)$ of

$$
(\sigma \nabla p, \nabla q)=-(\sigma \psi, \nabla q) \text {. }
$$

We now apply the same transformation to the right-hand side of (19) as in Theorem 2 to show that $p$ is actually solution to

$$
(\sigma \nabla p, \nabla q)=-\sum_{F \in \mathcal{F}}[[\sigma]]_{F}\left(\boldsymbol{\psi} \cdot \mathbf{n}_{F}, q\right)_{F} \quad \forall q \in H_{0}^{1}(\Omega),
$$

and it follows (see subsection 1.3) that $\mathbf{R} \in \mathbf{P} \mathbf{H}^{s}(\Omega, \mathscr{P})$ and

$$
\sum_{k=1}^{S}|\mathbf{R}|_{s, \Omega_{k}} \lesssim \sum_{F \in \mathcal{F}}\|\boldsymbol{\psi}\|_{1 / 2, F} \lesssim\|\boldsymbol{\psi}\|_{1}
$$

for all $0<s<\tau(\sigma)$.

Then (16) follows since $\nabla \times \mathbf{H}=\sigma \mathbf{R}$ and $\sigma$ is constant on each $\Omega_{k} \in \mathscr{P}$ by construction.

Finally, we also have (17) since $\mathbf{H}^{t}(\Omega)=\mathbf{P} \mathbf{H}^{t}(\Omega, \mathscr{P})$ for all $t<\tilde{\tau}(\sigma) \leq \tau(\sigma)$.

\section{Finite element discretization.}

2.1. Settings. The FE spaces we use consist of the standard Nédélec's edge elements [36]. Because we work with low-regularity fields, we will employ special "quasi-interpolation" operators defined in [20]. These operators are rigorously analyzed for meshes obtained from a single reference element through linear mappings. In particular, meshes made of tetrahedra or cubes are covered. For further discussions on quasi-interpolation operators, we refer the reader to $[28,20,21,43]$.

We introduce a family of tetrahedral (or cubic) meshes $\mathcal{T}_{h}=\{K\}$ of $\Omega$, with parameter $h>0$. We assume that this family of meshes is regular in the sense of [14]. Every mesh is conforming, which means that the intersection of two cells $\overline{K_{+}} \cap \overline{K_{-}}$is either a full face, a full edge, or a node of the two cells. Additionally, each cell $K \in \mathcal{T}_{h}$ is a "shape regular" tetrahedron, i.e., it satisfies

$$
\operatorname{diam} K \leq h, \quad \frac{\rho(K)}{\operatorname{diam} K} \geq \beta,
$$

where $\beta \in(0,1)$ is an $h$-independent constant and

$$
\rho(K)=\sup \{r>0 \mid \exists \mathbf{x} \in K, B(\mathbf{x}, r) \subset K\}, \quad \operatorname{diam}(K)=\sup \{|\mathbf{x}-\mathbf{y}| \mid \mathbf{x}, \mathbf{y} \in K\} .
$$


We say that a mesh $\mathcal{T}_{h}$ is a $\mathscr{P}$-fitting mesh (or simply, a fitting mesh) if

$$
\forall K \in \mathcal{T}_{h}, \exists \Omega_{k} \in \mathscr{P} \text { such that } K \subset \Omega_{k} .
$$

On the other hand, a mesh that does not satisfy (21) will be called a non- $\mathscr{P}$-fitting mesh (or simply, a nonfitting mesh).

For a given $h>0$, the space of first order Nédélec's element space is defined in $[36]$ as

$$
\mathbf{V}_{h}=\left\{\mathbf{v}_{h} \in H(\operatorname{curl}, \Omega)\left|\mathbf{v}_{h}\right|_{K} \in \mathcal{R}(K) \quad \forall K \in \mathcal{T}_{h}\right\}
$$

where

$$
\mathcal{R}(K)=\left\{\mathbf{v} \in \mathcal{P}_{1}(K)^{3} \mid \nabla \mathbf{v}+(\nabla \mathbf{v})^{T}=0\right\} .
$$

While the space $\mathbf{V}_{h}$ is actually used to approximate the magnetic field, it misses the essential boundary conditions of the electric field. Thus, in order to approximate the electric field, we further introduce

$$
\mathbf{V}_{h, 0}=\mathbf{V}_{h} \cap \mathbf{H}_{0}(\operatorname{curl}, \Omega)=\left\{\mathbf{v}_{h} \in \mathbf{V}_{h} \mid \mathbf{v}_{h} \times \mathbf{n}=0 \text { on } \partial \Omega\right\} .
$$

We also introduce the space $S^{h}$ of Lagrange FEs, defined by

$$
S^{h}=\left\{q^{h} \in H^{1}(\Omega)\left|q^{h}\right|_{K} \in \mathcal{P}_{1}(K) \quad \forall K \in \mathcal{T}_{h}\right\}
$$

and

$$
S_{0}^{h}=S^{h} \cap H_{0}^{1}(\Omega)=\left\{q^{h} \in S^{h} \mid q^{h}=0 \text { on } \partial \Omega\right\} .
$$

It is easily seen that if $q^{h} \in S^{h}$ (resp., if $q^{h} \in S_{0}^{h}$ ), then $\nabla q^{h} \in \mathbf{V}_{h}$ (resp. , $\nabla q^{h} \in \mathbf{V}_{h, 0}$ ).

For sufficiently regular functions $\mathbf{v}: \Omega \rightarrow \mathbb{C}^{3}$ and $q: \Omega \rightarrow \mathbb{C}$, it is customary to introduce the edge and the nodal interpolant $\Pi_{h}^{N} \mathbf{v} \in \mathbf{V}_{h}$ and $r^{h, N}(q) \in S^{h}$.

The edge interpolant was first designed by Nédélec [36] for regular fields $\mathbf{v} \in$ $\mathbf{H}^{2}(\Omega)$. As pointed out in section 4.1 of [28], the definition can be extended for fitting meshes as soon as $\mathbf{v} \in \mathbf{P} H^{s}(\Omega, \mathscr{P})$ and $\nabla \times \mathbf{v} \in \mathbf{P} \mathbf{H}^{s}(\Omega, \mathscr{P})$ with $s>1 / 2$.

Similarly, the nodal interpolant $r^{h, N}(q) \in S^{h}$ requires the function $q$ to be continuous [14], and as a result it is well defined if $q \in H^{1}(\Omega) \cap P H^{1+s}(\Omega, \mathscr{P})$ with $s>1 / 2$.

In addition, there exist interpolants $\Pi_{h, 0}^{N} \mathbf{v} \in \mathbf{V}_{h, 0}$ and $r_{0}^{h, N}(q)$ that incorporate essential boundary conditions and are well defined under the same conditions as $\Pi_{h}^{N}$ and $r^{h, N}$.

Since we work with low regularity fields, the aforementioned interpolation operators are insufficient. Hence, we will also employ "quasi-interpolation" operators which are well defined when $\mathbf{v} \in \mathbf{L}^{2}(\Omega)$ and $q \in L^{2}(\Omega)$. Specifically we will use the operators $\Pi_{h}^{Q}: \mathbf{L}^{2}(\Omega) \rightarrow \mathbf{V}_{h}, \Pi_{h, 0}^{Q}: \mathbf{L}^{2}(\Omega) \rightarrow \mathbf{V}_{h, 0}, r^{h, Q}: L^{2}(\Omega) \rightarrow S^{h}$ and $r_{0}^{h, Q}: L^{2}(\Omega) \rightarrow S_{0}^{h}$ that are defined in sections 5 and 6 of [20].

For nonfitting meshes, we employ the quasi-interpolation operators. For fitting meshes, we employ the quasi-interpolation operators when $\tau(\sigma) \leq 1 / 2$, and the standard Nédélec and Lagrange interpolants otherwise. We summarize the results we shall require in Propositions 5 and 6.

Proposition 5. For all $\mathbf{v} \in \mathbf{L}^{2}(\Omega)$, there exists an element $\Pi_{h, 0}^{Q} \mathbf{v} \in \mathbf{V}_{h, 0}$ such that 
- if $\mathbf{v} \in \mathbf{H}^{t}(\Omega)$ for some $t \in[0,1]$, then it holds that

$$
\left\|\mathbf{v}-\Pi_{h, 0}^{Q} \mathbf{v}\right\|_{0} \lesssim h^{t}|\mathbf{v}|_{t}
$$

- if $\nabla \times \mathbf{v} \in \mathbf{H}^{t}(\Omega)$ for some $t \in[0,1]$, we have

$$
\left\|\nabla \times\left(\mathbf{v}-\Pi_{h, 0}^{Q} \mathbf{v}\right)\right\|_{0} \lesssim h^{t}|\nabla \times \mathbf{v}|_{t}
$$

- if $\mathbf{v}=\nabla q$ for some $q \in H_{0}^{1}(\Omega)$, then there exists an element $r_{0}^{h, Q}(q) \in S_{0}^{h}$ such that

$$
\Pi_{h, 0}^{Q}(\nabla q)=\nabla r_{0}^{h, Q}(q) .
$$

In addition, if $\mathcal{T}_{h}$ is a $\mathscr{P}$-fitting mesh and if $\mathbf{v} \in \mathbf{P} \mathbf{H}^{s}(\Omega, \mathscr{P})$ with $s>1 / 2$, then there exists an element $\Pi_{h, 0}^{N} \mathbf{v} \in \mathbf{V}_{h, 0}$ such that

- we have

$$
\left\|\mathbf{v}-\Pi_{h, 0}^{N} \mathbf{v}\right\|_{0} \lesssim h^{s} \sum_{k=1}^{S}|\mathbf{v}|_{s, \Omega_{k}}
$$

- if $\nabla \times \mathbf{v} \in \mathbf{P H}^{t}(\Omega)$ for some $t \in(1 / 2,1]$, then

$$
\left\|\nabla \times\left(\mathbf{v}-\Pi_{h, 0}^{N} \mathbf{v}\right)\right\|_{0} \lesssim h^{t} \sum_{k=1}^{S}|\nabla \times \mathbf{v}|_{t, \Omega_{k}}
$$

- if $\mathbf{v}=\nabla q$ for some $q \in H_{0}^{1}(\Omega) \cap H^{1+s}(\Omega)$, then there exists an element $r_{0}^{h, N}(q) \in S_{0}^{h}$ such that

$$
\Pi_{h, 0}^{N}(\nabla q)=\nabla r_{0}^{h, N}(q)
$$

If $\mathcal{T}_{h}$ is a nonfitting mesh, we define the interpolation operators as $\Pi_{h, 0}=\Pi_{h, 0}^{Q}$ and $r_{0}^{h}=r_{0}^{h, Q}$. For a fitting mesh $\mathcal{T}_{h}$, we set $\Pi_{h, 0}=\Pi_{h, 0}^{Q}$ and $r_{0}^{h}=r_{0}^{h, Q}$ when $\tau(\sigma) \leq 1 / 2$, and $\Pi_{h, 0}=\Pi_{h, 0}^{N}$ and $r_{0}^{h}=r_{0}^{h, N}$ otherwise.

Proof. Interpolation estimate (22) is a direct consequence of Theorem 6.4 of [20]. To establish (23), we require the interpolation operator $R_{h, 0}^{Q}: \mathbf{L}^{2}(\Omega) \rightarrow \mathbf{W}_{h, 0} \subset$ $\mathbf{H}_{0}(\operatorname{div}, \Omega)$, where $\mathbf{W}_{h, 0}$ is the space of first order Raviart-Thomas elements, that is introduced in section 6 of [20]. Then, for $\mathbf{v} \in \mathbf{H}_{0}(\operatorname{curl}, \Omega)$ with $\nabla \times \mathbf{v} \in \mathbf{H}^{t}(\Omega)$, we have

$$
\nabla \times\left(\mathbf{v}-\Pi_{h, 0}^{Q} \mathbf{v}\right)=\nabla \times \mathbf{v}-R_{h, 0}^{Q}(\nabla \times \mathbf{v}),
$$

and by applying Theorem 6.4 of [20], we obtain that

$$
\left\|\nabla \times \mathbf{v}-R_{h, 0}^{Q}(\nabla \times \mathbf{v})\right\|_{0} \lesssim h^{t}|\nabla \times \mathbf{v}|_{t} .
$$

On the other hand, the operators $\Pi_{h, 0}^{N}$ and $r_{0}^{h, N}$, respectively, correspond to the standard Nédélec's edge and Lagrange nodal interpolants. These are well defined under the assumed regularity, and interpolation error estimates (24) and (25) follow from standard arguments (see, for instance, [29]). 
In Proposition 6, we introduce the quasi-interpolation operator $\Pi_{h}$, which is the analogue of $\Pi_{h, 0}$ for the space $\mathbf{V}_{h}$.

Proposition 6. For all $\mathbf{v} \in \mathbf{L}^{2}(\Omega)$, there exists an element $\Pi_{h}^{Q} \mathbf{v} \in \mathbf{V}_{h}$ such that

- if $\mathbf{v} \in \mathbf{H}^{t}(\Omega)$ for some $t \in[0,1]$, then it holds that

$$
\left\|\mathbf{v}-\Pi_{h}^{Q} \mathbf{v}\right\|_{0} \lesssim h^{t}|\mathbf{v}|_{t}
$$

- if $\nabla \times \mathbf{v} \in \mathbf{H}^{t}(\Omega)$ for some $t \in[0,1]$, we have

$$
\left\|\nabla \times\left(\mathbf{v}-\Pi_{h}^{Q} \mathbf{v}\right)\right\|_{0} \lesssim h^{t}|\nabla \times \mathbf{v}|_{t}
$$

- if $\mathbf{v}=\nabla q$ for some $q \in H^{1}(\Omega)$, then there exists an element $r^{h, Q}(q) \in S^{h}$ such that

$$
\Pi_{h}^{Q}(\nabla q)=\nabla r^{h, Q}(q)
$$

In addition, if $\mathcal{T}_{h}$ is a $\mathscr{P}$-fitting mesh and if $\mathbf{v} \in \mathbf{P} \mathbf{H}^{s}(\Omega, \mathscr{P})$ with $s>1 / 2$, then there exists an element $\Pi_{h}^{N} \mathbf{v} \in \mathbf{V}_{h}$ such that

- we have

$$
\left\|\mathbf{v}-\Pi_{h}^{N} \mathbf{v}\right\|_{0} \lesssim h^{s} \sum_{k=1}^{S}|\mathbf{v}|_{s, \Omega_{k}}
$$

- if $\nabla \times \mathbf{v} \in \mathbf{P H}^{t}(\Omega)$ for some $t \in[0,1]$, then

$$
\left\|\nabla \times\left(\mathbf{v}-\Pi_{h}^{N} \mathbf{v}\right)\right\|_{0} \lesssim h^{t} \sum_{k=1}^{S}|\nabla \times \mathbf{v}|_{t, \Omega_{k}} ;
$$

- if $\mathbf{v}=\nabla q$ for some $q \in H^{1}(\Omega) \cap H^{1+s}(\Omega)$, then there exists an element $r^{h, N}(q) \in S^{h}$ such that

$$
\Pi_{h}^{N}(\nabla q)=\nabla r^{h, N}(q)
$$

If $\mathcal{T}_{h}$ is a nonfitting mesh, we define the interpolation operators as $\Pi_{h}=\Pi_{h}^{Q}$ and $r^{h}=r^{h, Q}$. For a fitting mesh $\mathcal{T}_{h}$, we set $\Pi_{h}=\Pi_{h}^{Q}$ and $r^{h}=r^{h, Q}$ when $\tau(\sigma) \leq 1 / 2$, and $\Pi_{h}=\Pi_{h}^{N}$ and $r^{h}=r^{h, N}$ otherwise.

Proof. The proof is similar to the proof of Proposition 5, using Theorem 5.2 of [20] instead of Theorem 6.4.

2.2. A discussion on fitting and nonfitting meshes. As depicted in Figure 1 , nonfitting meshes have interesting applications, as they can simplify implementations and/or reduce computational costs. Unfortunately, they also come with additional difficulties that we shall discuss in this subsection. These complications occur because material discontinuities are allowed inside mesh cells. As a result, the normal component of the electric field jumps through the interfaces inside mesh cells, so that a discontinuous function must be approximated by continuous FE shape functions. Also, numerical integration becomes more challenging.

We will describe these two issues and explain precisely what are the implications and the possible solutions. For simplicity, we only analyze the electric field formulation in this discussion. However, all our remarks remain unchanged for the magnetic field formulation. 
2.2.1. Linear system assembling. The $\mathrm{FE}$ linear system associated with variational formulation (4) has entries given by $M_{i j}=b_{\mathbf{E}}\left(\phi_{j}, \phi_{i}\right)$, where $\phi_{i}, \phi_{j} \in \mathbf{V}_{h, 0}$ are $\mathrm{FE}$ shape functions. In particular, it is required to compute the quantities

$$
\int_{K} \sigma \phi_{j} \cdot \phi_{i}
$$

where $K$ is a mesh cell. When a fitting mesh is used, $\sigma$ takes one constant value $\sigma_{K}$ on $K$, so that computing (30) amounts to integrating a polynomial function onto a tetrahedron. This can be done analytically, and optimized techniques using affine mappings can be used to do it efficiently $[14,34]$. When the mesh does not fit the conductivity model, additional techniques are required [11]. Fortunately, as shown in [13], an efficient and accurate technique has been developed. Briefly speaking, the key idea is to introduce an approximation $\sigma_{\epsilon}$ to $\sigma$ such that

$$
\int_{K} \sigma_{\epsilon} \phi_{j} \cdot \phi_{i}
$$

is easily computed and is a good approximation to (30). Specifically, we partition the reference cell $\hat{K}$ into a submesh $\hat{\mathcal{T}}_{\epsilon}$. For each cell $K, \sigma$ is approximated by

$$
\left.\sigma_{\epsilon}\right|_{K}=\sum_{A \in \hat{\mathcal{T}}_{\epsilon}} \sigma\left(\mathcal{F}_{K}\left(\mathbf{x}_{A}\right)\right) \mathbf{1}_{\mathcal{F}_{K}(A)},
$$

where $\mathbf{x}_{A}$ denotes the center $A$ and $\mathcal{F}_{K}$ is the linear map between $\hat{K}$ and $K$. If $\sigma$ is discontinuous at $\mathcal{F}\left(\mathbf{x}_{A}\right)$, the minimum neighboring value is taken for $\sigma\left(\mathcal{F}\left(\mathbf{x}_{A}\right)\right)$. Figure 2 illustrates this process. Thanks to this definition, we can compute (31) efficiently based on the precomputed integrals

$$
I_{n m}^{k l}(A)=\int_{A} \hat{\psi}_{n}^{k} \hat{\psi}_{m}^{l} \quad \forall A \in \hat{\mathcal{T}}_{\epsilon},
$$

where $\hat{\psi}^{k}$ and $\hat{\psi}^{l}$ are the shape functions associated with the reference element $\hat{K}$.

2.2.2. Convergence rates. The second problem is due to the lack of regularity of the solution inside the mesh cells. In terms of Sobolev spaces, the accuracy of the FE solution is directly related to the regularity of the solution inside each cell.

If the mesh is $\mathcal{P}$-fitting, then each cell $K \in \mathcal{T}_{h}$ is included inside one subdomain $\Omega_{k} \in \mathscr{P}$. As a result, $K \subset \Omega_{k}$, and $\mathbf{E} \in \mathbf{P} \mathbf{H}^{s}(\Omega, \mathscr{P})$ implies that $\left.\mathbf{E}\right|_{K} \in \mathbf{H}^{s}(K)$ for all $0<s<\tau(\sigma)$. On the other hand, if the mesh does not fit the partition $\mathscr{P}$, then we only have $\left.\mathbf{E}\right|_{K} \in \mathbf{H}^{t}(K)$ for $0<t<\tilde{\tau}(\sigma)=\min (\tau(\sigma), 1 / 2)$. Thus, in the absence of singularity or, more generally, if $\tau(\sigma)>1 / 2$, we cannot expect nonfitting meshes to be as accurate as fitting meshes, because the electric field is less regular inside each cell. Indeed, we will show that the convergence rate of the electric field approximation in the $L^{2}$-norm is $\mathcal{O}\left(h^{\tau(\sigma)}\right)$ for fitting meshes and only $\mathcal{O}\left(h^{\tilde{\tau}(\sigma)}\right)$ for nonfitting meshes.

In order to illustrate this point, let us consider the case of a cubic domain with plane horizontal interfaces. Then according to [17], we have $\mathbf{E} \in \mathbf{P H}^{1}(\Omega, \mathscr{P})$ and, if $\mathcal{T}_{h}$ is a fitting mesh, then $\left.\mathbf{E}\right|_{K} \in \mathbf{H}^{1}(K)$ for all $K \in \mathcal{T}_{h}$. It follows that the convergence rate of Nédélec's FE will be linear if fitting meshes are used. However, for a nonfitting mesh, we only have $\left.\mathbf{E}\right|_{K} \in \mathbf{H}^{(1-\delta) / 2}(K)$, since the vertical component of $\mathbf{E}$ might jump inside $K$. (Recalling subsection $1.1, \delta>0$ is an arbitrarily small constant.) As a result, the convergence rate of Nédélec's FEs with nonfitting meshes will be at most $\mathcal{O}\left(h^{(1-\delta) / 2}\right)$. 

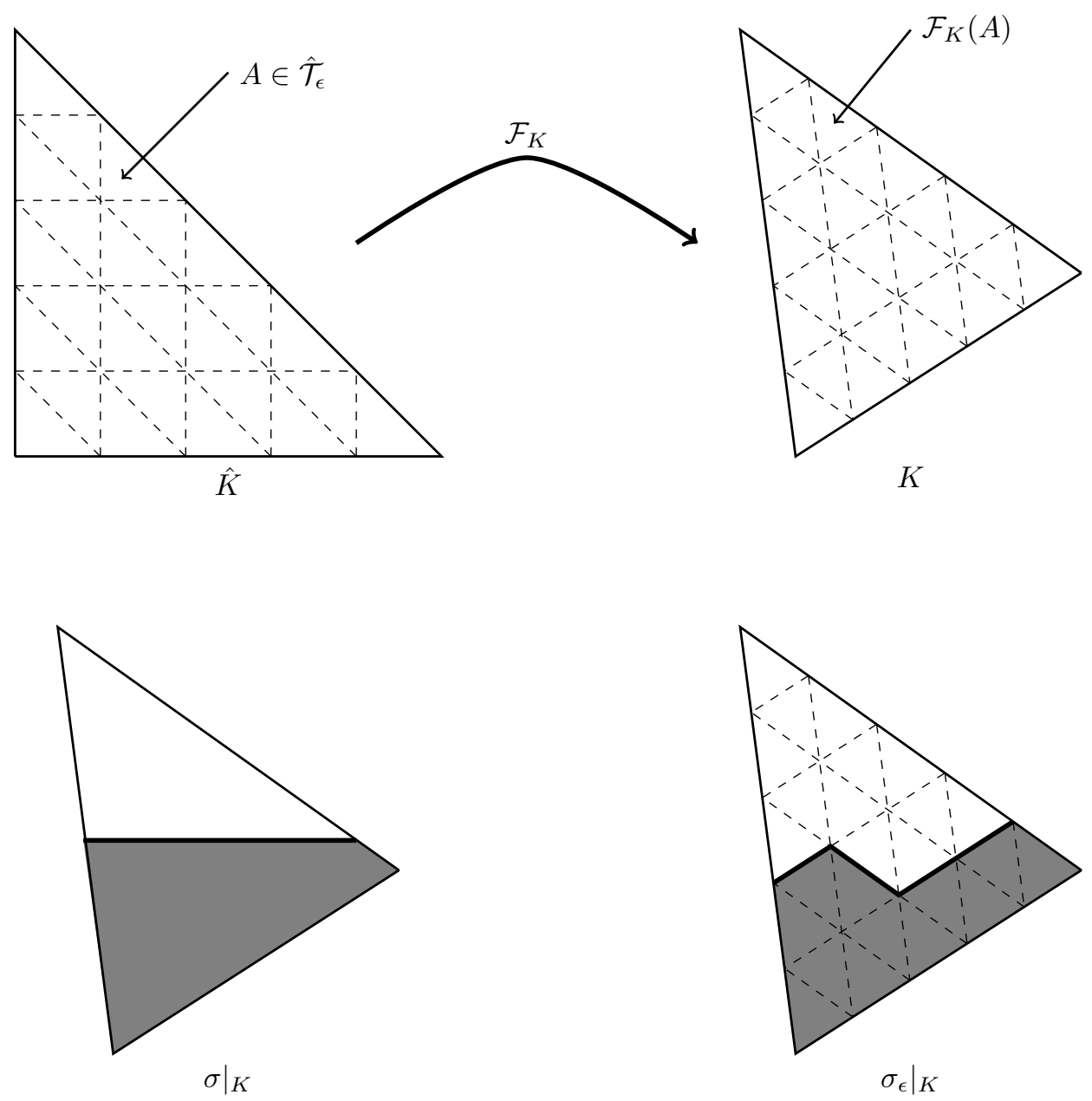

FIG. 2. Construction of $\sigma_{\epsilon}$.

The main novelty of our analysis is that when the permeability is constant, the approximations of the electric and magnetic fields have different convergence rates. Indeed, we are able to show that if the electric field converges as $\mathcal{O}\left(h^{s}\right)$, then the magnetic field error decreases as $\mathcal{O}\left(h^{\min (1,2 s)}\right)$. For a cubic domain with horizontal layers, it means that the magnetic field approximation on a nonfitting mesh will converge as $\mathcal{O}\left(h^{(1-\delta)}\right)$. As a result, the observed convergence rate is linear, just as in the case of fitting meshes. More generally, it implies that fitting and nonfitting meshes will provide the same convergence rate for the magnetic field error in the $L^{2}$-norm: $\mathcal{O}\left(h^{\min (1,2 s)}\right)$, where $0<s<\tau(\sigma)$.

Thus, our main claim is that if nonfitting meshes do imply an accuracy loss concerning the electric field approximation, they provide the same convergence rate as fitting meshes for the magnetic field approximation. In the following subsections, we support this claim with rigorous proofs, both for the electric and magnetic field formulations of Maxwell's equations. 
2.3. The electric field formulation. We first discretize the electric field formulation (4). In this case, we seek a Galerkin approximation $\mathbf{E}_{h}$ to $\mathbf{E}$ in the space $\mathbf{V}_{h, 0}$. As usual, the approximation is defined using the discrete variational problem: Find $\mathbf{E}_{h} \in \mathbf{V}_{h, 0}$ such that

$$
b_{\mathbf{E}}\left(\mathbf{E}_{h}, \mathbf{v}_{h}\right)=i \omega \mu_{0}\left(\mathbf{J}, \mathbf{v}_{h}\right)-\left(\mathbf{M}, \nabla \times \mathbf{v}_{h}\right) \quad \forall \mathbf{v}_{h} \in \mathbf{V}_{h, 0} .
$$

Solving (32) amounts to factorizing a linear system. Then, the discrete representation of the magnetic field $\mathbf{H}_{h}$ is obtained by postprocessing as

$$
\mathbf{H}_{h}=\frac{1}{i \omega \mu_{0}}\left(\mathbf{M}+\nabla \times \mathbf{E}_{h}\right) .
$$

From (33), it is clear that the error committed on the approximation of the magnetic field in the $L^{2}$-norm is proportional to $\left|\nabla \times\left(\mathbf{E}-\mathbf{E}_{h}\right)\right|_{0}$. Thus, we will speak of "magnetic field" error to denote $\left|\nabla \times\left(\mathbf{E}-\mathbf{E}_{h}\right)\right|_{0}$. On the other hand, we will name "electric field" error the quantity $\left|\mathbf{E}-\mathbf{E}_{h}\right|_{0}$.

In view of Theorem 2, throughout this subsection we require that $\mathbf{J} \in \mathbf{H}(\operatorname{div}, \Omega)$ and $\mathbf{M} \in \mathbf{H}(\operatorname{curl}, \Omega)$.

2.3.1. Interpolation error estimates. In order to simplify the following proofs, let us define, for arbitrary $\mathbf{j}, \mathbf{m} \in \mathbf{L}^{2}(\Omega), \mathcal{E}(\mathbf{j}, \mathbf{m}) \in \mathbf{H}_{0}($ curl, $\Omega)$ as the solution to

$$
b_{\mathbf{E}}(\mathcal{E}(\mathbf{j}, \mathbf{m}), \mathbf{v})=i \omega \mu_{0}(\mathbf{j}, \mathbf{v})-(\mathbf{m}, \nabla \times \mathbf{v}) \quad \forall \mathbf{v} \in \mathbf{H}_{0}(\operatorname{curl}, \Omega) .
$$

In particular, $\mathbf{E}=\mathcal{E}(\mathbf{J}, \mathbf{M})$, and Theorems 1 and 2 hold if $\mathbf{E}, \mathbf{J}$, and $\mathbf{M}$ are replaced by $\mathcal{E}(\mathbf{j}, \mathbf{m}), \mathbf{j}$, and $\mathbf{m}$, respectively.

Later, we will need to quantify, for arbitrary right-hand sides $\mathbf{j}, \mathbf{m} \in \mathbf{L}^{2}(\Omega)$ the ability of the interpolant $\Pi_{h, 0} \mathcal{E}(\mathbf{j}, \mathbf{m})$ to approximate the solution $\mathcal{E}(\mathbf{j}, \mathbf{m})$. Hence, we introduce for $h>0$

$$
\eta_{h}^{\mathbf{E}}=\sup _{\mathbf{j} \in \mathbf{H}(d i v, \Omega), \mathbf{m} \in \mathbf{L}^{2}(\Omega)} \frac{\left|\mathcal{E}(\mathbf{j}, \mathbf{m})-\Pi_{h, 0} \mathcal{E}(\mathbf{j}, \mathbf{m})\right|_{0}}{\|\mathbf{j}\|_{d i v}+|\mathbf{m}|_{0}}
$$

and

$$
\eta_{h}^{\mathbf{H}}=\sup _{\mathbf{j} \in \mathbf{L}^{2}(\Omega), \mathbf{m} \in \mathbf{H}(\operatorname{curl}, \Omega)} \frac{\left|\nabla \times\left(\mathcal{E}(\mathbf{j}, \mathbf{m})-\Pi_{h, 0} \mathcal{E}(\mathbf{j}, \mathbf{m})\right)\right|_{0}}{|\mathbf{j}|_{0}+\|\mathbf{m}\|_{\text {curl }}} .
$$

The quantities $\eta_{h}^{\mathbf{E}}$ and $\eta_{h}^{\mathbf{H}}$ control the interpolation error on the electric and magnetic fields, respectively, since by definition, we have

$$
\left|\mathcal{E}(\mathbf{j}, \mathbf{m})-\Pi_{h, 0} \mathcal{E}(\mathbf{j}, \mathbf{m})\right|_{0} \lesssim \eta_{h}^{\mathbf{E}}\left(\|\mathbf{j}\|_{d i v}+|\mathbf{m}|_{0},\right) \quad \forall \mathbf{j} \in \mathbf{H}(\operatorname{div}, \Omega), \forall \mathbf{m} \in \mathbf{L}^{2}(\Omega)
$$

and

$$
\left|\nabla \times\left(\mathcal{E}(\mathbf{j}, \mathbf{m})-\Pi_{h, 0} \mathcal{E}(\mathbf{j}, \mathbf{m})\right)\right|_{0} \lesssim \eta_{h}^{\mathbf{E}}\left(|\mathbf{j}|_{0}+\|\mathbf{m}\|_{\text {curl }}\right) \quad \forall \mathbf{j} \in \mathbf{L}^{2}(\Omega), \forall \mathbf{m} \in \mathbf{H}(\text { curl }, \Omega)
$$

We point out that the conditions $\mathbf{j} \in \mathbf{H}(\operatorname{div}, \Omega)$ and $\mathbf{m} \in \mathbf{H}(\operatorname{curl}, \Omega)$ are "built in" the definitions of $\eta_{h}^{\mathbf{E}}$ and $\eta_{h}^{\mathbf{H}}$, respectively.

In the following theorem, we bound the quantities $\eta_{h}^{\mathbf{E}}$ and $\eta_{h}^{\mathbf{H}}$. We observe that the $\mathscr{P}$-fitting property impacts the behavior of $\eta_{h}^{\mathbf{E}}$ but not that of $\eta_{h}^{\mathbf{H}}$. 
TheOREM 7. It holds that

$$
\eta_{h}^{\mathbf{H}} \lesssim h,
$$

whether or not the mesh is $\mathscr{P}$-fitting.

If $\mathcal{T}_{h}$ is a $\mathscr{P}_{\text {-fitting mesh, then we have }}$

$$
\eta_{h}^{\mathbf{E}} \lesssim h^{s}
$$

for all $0<s<\tau(\sigma)$.

If $\tilde{\mathcal{T}}_{h}$ is a non- $\mathscr{P}$-fitting mesh, we only have

$$
\eta_{h}^{\mathbf{E}} \lesssim h^{t}
$$

for all $0<t<\tilde{\tau}(\sigma)$.

Proof. The proof is a direct combination of Theorem 2 and Proposition 5.

2.3.2. Electric field error estimates. According to Theorem 7, we see from (34), (35), and (36) that the interpolation error in the $L^{2}$-norm is smaller for the magnetic field than for the electric field. Unfortunately, as shown in Theorem 8, a direct application of Céa's lemma gives the same convergence rate for both electromagnetic fields. Hence, the improved accuracy of the interpolant for the magnetic field stays hidden.

Theorem 8. For all $h>0$, there exists a unique $\mathbf{E}_{h} \in \mathbf{V}_{h, 0}$ solution to (32). Furthermore, the discrete solution $\mathbf{E}_{h}$ satisfies

$$
\left\|\mathbf{E}-\mathbf{E}_{h}\right\|_{\text {curl }} \lesssim\left(\eta_{h}^{\mathbf{E}}+\eta_{h}^{\mathbf{H}}\right)\left(\|\mathbf{J}\|_{\text {div }}+\|\mathbf{M}\|_{\text {curl }}\right) .
$$

Proof. Since $b_{\mathbf{E}}$ is coercive, the proof is a simple application of Céa's lemma. Indeed, by Galerkin orthogonality, we have

$$
\begin{aligned}
\left\|\mathbf{E}-\mathbf{E}_{h}\right\|_{c u r l}^{2} & \lesssim\left|b_{\mathbf{E}}\left(\mathbf{E}-\mathbf{E}_{h}, \mathbf{E}-\mathbf{E}_{h}\right)\right| \\
& =\left|b_{\mathbf{E}}\left(\mathbf{E}-\mathbf{E}_{h}, \mathbf{E}-\Pi_{h, 0} \mathbf{E}\right)\right| \\
& \lesssim\left\|\mathbf{E}-\mathbf{E}_{h}\right\|_{\text {curl }}\left\|\mathbf{E}-\Pi_{h, 0} \mathbf{E}\right\|_{\text {curl }} .
\end{aligned}
$$

Then, we have

$$
\begin{aligned}
\left\|\mathbf{E}-\Pi_{h, 0} \mathbf{E}\right\|_{\text {curl }} & =\left|\mathcal{E}(\mathbf{J}, \mathbf{M})-\Pi_{h, 0} \mathcal{E}(\mathbf{J}, \mathbf{M})\right|_{0}+\left|\nabla \times\left(\mathcal{E}(\mathbf{J}, \mathbf{M})-\Pi_{h, 0} \mathcal{E}(\mathbf{J}, \mathbf{M})\right)\right|_{0} \\
& \lesssim \eta_{h}^{\mathbf{E}}\left(\|\mathbf{J}\|_{\text {div }}+|\mathbf{M}|_{0}\right)+\eta_{h}^{\mathbf{H}}\left(|\mathbf{J}|_{0}+\|\mathbf{M}\|_{\text {curl }}\right) \\
& \lesssim\left(\eta_{h}^{\mathbf{E}}+\eta_{h}^{\mathbf{H}}\right)\left(\|\mathbf{J}\|_{\text {div }}+\|\mathbf{M}\|_{\text {curl }}\right)
\end{aligned}
$$

and (37) follows.

As pointed out in [33], once the interpolation error estimates are established, Céa's lemma trivially implies the convergence of Nédélec's FEs when the conductivity is strictly positive. In [33], Céa's lemma is used exactly as in Theorem 8, with the notable difference that the conductivity $\sigma$ is assumed to be continuous, and the solution $\mathbf{E} \in \mathbf{H}^{2}(\Omega)$. In this case, we immediately have $\eta_{h}^{\mathbf{E}} \simeq \eta_{h}^{\mathbf{H}} \simeq h$, so that Céa's lemma directly yields optimal convergence rates for both the electric and magnetic fields.

In [33], Monk also considers the more difficult case where $\sigma=0$ and $\varepsilon>0$. Then, the sesquilinear form is no longer coercive, and special duality techniques have to be employed to prove the well-posedness and convergence in some asymptotic range. 
Then again, the solution is assumed to be regular, so that the final error estimate is linear in the full $H$ (curl)-norm.

Generally speaking, when the solution is regular, it makes sense to measure FE errors in the full $H(\mathrm{curl})$-norm, as its two components converge at the same rate: linearly. Also, in contrast with the Poisson problem, there is no need to use duality techniques à la Aubin and Nitsche, since the result given by Céa's lemma is already optimal.

In the case of heterogeneous media with discontinuous permittivity and permeability, both electromagnetic fields have low regularity, and it makes sense to derive error estimates in the full $H(\mathrm{curl})$-norm as well [34, 28]. The resulting convergence rate then corresponds to the lowest regularity of the two fields.

Here, since the magnetic field is always regular, we feel that it is important to distinguish the convergence rate of the two components of the $H($ curl)-norm, that is, the $L^{2}$-norm of the electric field, and the $L^{2}$-norm of the magnetic field. We first establish the electric field error estimate in Corollary 9. The magnetic field error estimate will be derived in Corollary 11 of the next subsection.

Since $\eta_{h}^{\mathbf{E}}+\eta_{h}^{\mathbf{H}} \simeq \eta_{h}^{\mathbf{E}}$, error estimate (37) is optimal for the electric field $L^{2}$ error. Indeed, it shows that the FE solution converges as $\eta_{h}^{\mathbf{E}}$, just as the interpolant, in terms of the electric field $L^{2}$ error. We record this result in the next corollary; its proof is a direct application of Theorems 7 and 8 .

Corollary 9. If $\mathcal{T}_{h}$ is a $\mathscr{P}$-fitting mesh, it holds that

$$
\left|\mathbf{E}-\mathbf{E}_{h}\right|_{0} \lesssim h^{s}\left(\|\mathbf{J}\|_{\text {div }}+\|\mathbf{M}\|_{\text {curl }}\right)
$$

for all $0<s<\tau(\sigma)$.

If $\tilde{\mathcal{T}}_{h}$ is not $\mathscr{P}$-fitting, then we only have

$$
\left|\mathbf{E}-\mathbf{E}_{h}\right|_{0} \lesssim h^{t}\left(\|\mathbf{J}\|_{\text {div }}+\|\mathbf{M}\|_{\text {curl }}\right)
$$

for all $0<t<\tilde{\tau}(\sigma)$.

2.3.3. Magnetic field error estimates. Corollary 9 provides an optimal error estimate for the electric field. However, if we apply directly Theorem 6 to estimate the magnetic field error in the $L^{2}$-norm, we obtain

$$
\left|\nabla \times\left(\mathbf{E}-\mathbf{E}_{h}\right)\right|_{0} \lesssim h^{s}\left(\|\mathbf{J}\|_{d i v}+\|\mathbf{M}\|_{c u r l}\right)
$$

for all $0<s<\tau(\sigma)$ (or $\tilde{\tau}(\sigma)$ for a nonfitting mesh). However, from estimate (34) of Theorem 7 , we know that the convergence rate of the interpolant is linear for the magnetic field in the sense that

$$
\left|\nabla \times\left(\mathbf{E}-\Pi_{h, 0} \mathbf{E}\right)\right|_{0} \lesssim h\left(\|\mathbf{J}\|_{d i v}+\|\mathbf{M}\|_{\text {curl }}\right) .
$$

As a result, it is unclear whether (40) is optimal. In the next theorem, we show that (40) is actually suboptimal and provide an improved error estimate for the magnetic field.

THEOREM 10. It holds that

$$
\left|\nabla \times\left(\mathbf{E}-\mathbf{E}_{h}\right)\right|_{0} \lesssim\left(\eta_{h}^{\mathbf{H}}+\left(\eta_{h}^{\mathbf{E}}+\eta_{h}^{\mathbf{H}}\right)^{2}\right)\left(\|\mathbf{J}\|_{\text {div }}+\|\mathbf{M}\|_{\text {curl }}\right) .
$$

Proof. The proof relies on the Aubin-Nitsche duality trick, which needs to be slightly modified for our purpose. We define $\phi \in \mathbf{H}_{0}(\mathrm{curl}, \Omega)$ as the unique solution 
of

$$
b_{\mathbf{E}}(\mathbf{v}, \phi)=\left(\nabla \times \mathbf{v}, \nabla \times\left(\mathbf{E}-\mathbf{E}_{h}\right)\right) \quad \forall \mathbf{v} \in \mathbf{H}_{0}(\text { curl }, \Omega) .
$$

It can be easily seen that $\phi=\overline{\mathcal{E}(\mathbf{j}, \mathbf{m})}$ with $\mathbf{j}=\mathbf{0}$ and $\mathbf{m}=\overline{\nabla \times\left(\mathbf{E}-\mathbf{E}_{h}\right)}$. Because $\mathbf{0}=\mathbf{j} \in \mathbf{H}(\operatorname{div}, \Omega)$, we have

$$
\left|\phi-\Pi_{h, 0} \phi\right|_{0} \leq \eta_{h}^{\mathbf{E}}|\mathbf{m}|_{0}=\eta_{h}^{\mathbf{E}}\left|\nabla \times\left(\mathbf{E}-\mathbf{E}_{h}\right)\right|_{0} .
$$

Since $\nabla \times \mathbf{E}_{h}$ is a discontinuous (piecewise constant) function, $\mathbf{m} \notin \mathbf{H}($ curl, $\Omega$ ), and we cannot bound $\left|\nabla \times\left(\phi-\Pi_{h, 0} \phi\right)\right|_{0}$ directly. Hence, we introduce a decomposition of $\phi$ as $\phi=\mathbf{E}-\mathbf{E}_{h}+\boldsymbol{\psi}$, with $\boldsymbol{\psi} \in \mathbf{H}_{0}\left(\right.$ curl, $\Omega$ ) (because $\mathbf{E}, \mathbf{E}_{h} \in \mathbf{H}_{0}($ curl,,$\Omega)$ ) the solution of

$$
b_{\mathbf{E}}(\mathbf{v}, \boldsymbol{\psi})=b_{\mathbf{E}}(\mathbf{v}, \boldsymbol{\phi})-b_{\mathbf{E}}\left(\mathbf{v}, \mathbf{E}-\mathbf{E}_{h}\right)=\left(\mathbf{v},-i \omega \mu_{0} \sigma\left(\mathbf{E}-\mathbf{E}_{h}\right)\right) \quad \forall \mathbf{v} \in \mathbf{H}_{0}(c u r l, \Omega) .
$$

This equivalently means that $\boldsymbol{\psi}=\overline{\mathcal{E}(\mathbf{j}, \mathbf{m})}$ with $\mathbf{j}=-i \omega \mu_{0} \sigma \overline{\left(\mathbf{E}-\mathbf{E}_{h}\right)}$ and $\mathbf{m}=\mathbf{0}$. Then, we have $\mathbf{0}=\mathbf{m} \in \mathbf{H}(\operatorname{curl}, \Omega)$, and it follows that

$$
\begin{aligned}
\left|\nabla \times\left(\boldsymbol{\phi}-\Pi_{h, 0} \boldsymbol{\phi}\right)\right|_{0} & \leq\left|\nabla \times\left(\left(\mathbf{E}-\mathbf{E}_{h}\right)-\Pi_{h, 0}\left(\mathbf{E}-\mathbf{E}_{h}\right)\right)\right|_{0}+\left|\nabla \times\left(\boldsymbol{\psi}-\Pi_{h, 0} \boldsymbol{\psi}\right)\right|_{0} \\
& \lesssim\left|\nabla \times\left(\mathbf{E}-\Pi_{h, 0} \mathbf{E}\right)\right|_{0}+\eta_{h}^{\mathbf{H}}\left|\sigma\left(\mathbf{E}-\mathbf{E}_{h}\right)\right|_{0} \\
& \lesssim\left|\nabla \times\left(\mathbf{E}-\Pi_{h, 0} \mathbf{E}\right)\right|_{0}+\eta_{h}^{\mathbf{H}}\left|\left(\mathbf{E}-\mathbf{E}_{h}\right)\right|_{0} .
\end{aligned}
$$

By definition of $\phi$ and by Galerkin orthogonality, we have

$$
\begin{aligned}
\left|\nabla \times\left(\mathbf{E}-\mathbf{E}_{h}\right)\right|_{0}^{2} & =b_{\mathbf{E}}\left(\mathbf{E}-\mathbf{E}_{h}, \boldsymbol{\phi}\right) \\
& =b_{\mathbf{E}}\left(\mathbf{E}-\mathbf{E}_{h}, \boldsymbol{\phi}-\Pi_{h, 0} \boldsymbol{\phi}\right) \\
& \lesssim\left|\mathbf{E}-\mathbf{E}_{h}\right|_{0}\left|\boldsymbol{\phi}-\Pi_{h, 0} \boldsymbol{\phi}\right|_{0}+\left|\nabla \times\left(\mathbf{E}-\mathbf{E}_{h}\right)\right|_{0}\left|\nabla \times\left(\boldsymbol{\phi}-\Pi_{h, 0} \boldsymbol{\phi}\right)\right|_{0} \\
& \lesssim \eta_{h}^{\mathbf{E}}\left|\mathbf{E}-\mathbf{E}_{h}\right|_{0}\left|\nabla \times\left(\mathbf{E}-\mathbf{E}_{h}\right)\right|_{0}+\left|\nabla \times\left(\mathbf{E}-\mathbf{E}_{h}\right)\right|_{0}\left|\nabla \times\left(\boldsymbol{\phi}-\Pi_{h, 0} \boldsymbol{\phi}\right)\right|_{0} .
\end{aligned}
$$

Simplifying by $\left|\nabla \times\left(\mathbf{E}-\mathbf{E}_{h}\right)\right|_{0}$, we conclude that

$$
\begin{aligned}
\left|\nabla \times\left(\mathbf{E}-\mathbf{E}_{h}\right)\right|_{0} & \lesssim \eta_{h}^{\mathbf{E}}\left|\mathbf{E}-\mathbf{E}_{h}\right|_{0}+\left|\nabla \times\left(\boldsymbol{\phi}-\Pi_{h, 0} \boldsymbol{\phi}\right)\right|_{0} \\
& \lesssim\left|\nabla \times\left(\mathbf{E}-\Pi_{h, 0} \mathbf{E}\right)\right|_{0}+\left(\eta_{h}^{\mathbf{E}}+\eta_{h}^{\mathbf{H}}\right)\left|\mathbf{E}-\mathbf{E}_{h}\right|_{0}
\end{aligned}
$$

and (41) follows since, from Theorems 7 and 8, we have

$$
\left|\nabla \times\left(\mathbf{E}-\Pi_{h, 0} \mathbf{E}\right)\right|_{0} \lesssim \eta_{h}^{\mathbf{H}}\left(\|\mathbf{J}\|_{\text {div }}+\|\mathbf{M}\|_{\text {curl }}\right)
$$

and

$$
\left|\mathbf{E}-\mathbf{E}_{h}\right|_{0} \lesssim\left(\eta_{h}^{\mathbf{E}}+\eta_{h}^{\mathbf{H}}\right)\left(\|\mathbf{J}\|_{\text {div }}+\|\mathbf{M}\|_{\text {curl }}\right) .
$$

In Corollary 11, we directly apply Theorem 10 to provide explicit convergence rates in terms of mesh size, for both fitting and nonfitting meshes. We see that fitting and nonfitting meshes deliver virtually the same convergence rates (up to an arbitrarily small constant $\delta$ ).

Corollary 11. Assume that $\tau(\sigma) \leq 1 / 2$. Then it holds that

$$
\left|\nabla \times\left(\mathbf{E}-\mathbf{E}_{h}\right)\right|_{0} \lesssim h^{2 s}\left(\|\mathbf{J}\|_{d i v}+\|\mathbf{M}\|_{c u r l}\right)
$$

for all $0<s<\tau(\sigma)$ for both fitting and nonfitting meshes. 


$$
\text { If } \tau(\sigma)>1 / 2 \text {, then we have }
$$

$$
\left|\nabla \times\left(\mathbf{E}-\mathbf{E}_{h}\right)\right|_{0} \lesssim h\left(\|\mathbf{J}\|_{d i v}+\|\mathbf{M}\|_{\text {curl }}\right)
$$

for fitting meshes and

$$
\left|\nabla \times\left(\mathbf{E}-\mathbf{E}_{h}\right)\right|_{0} \lesssim h^{1-\delta}\left(\|\mathbf{J}\|_{d i v}+\|\mathbf{M}\|_{c u r l}\right)
$$

for nonfitting meshes.

Proof. The proof simply relies on applying the bounds of Theorem 7 to the factor $\eta_{h}^{\mathbf{H}}+\left(\eta_{h}^{\mathbf{E}}+\eta_{h}^{\mathbf{H}}\right)^{2}$ of (41).

2.4. The magnetic field formulation. We now consider the discretization of the magnetic field formulation (5) using Nédélec's edge elements. The discrete magnetic field is sought as the element $\mathbf{H}_{h} \in \mathbf{V}_{h}$ satisfying

$$
b_{\mathbf{H}}\left(\mathbf{H}_{h}, \mathbf{v}_{h}\right)=\left(\mathbf{M}, \mathbf{v}_{h}\right)+\left(\sigma^{-1} \mathbf{J}, \nabla \times \mathbf{v}_{h}\right) \quad \forall \mathbf{v}_{h} \in \mathbf{V}_{h}
$$

can be computed as $\tilde{\mathbf{E}}_{h}=\sigma^{-1}\left(\mathbf{J}-\nabla \times \mathbf{H}_{h}\right)$, and the error on the electric field is given by

$$
\left|\mathbf{E}-\tilde{\mathbf{E}}_{h}\right|_{0}=\left|\sigma^{-1}\left(\nabla \times \mathbf{H}-\nabla \times \mathbf{H}_{h}\right)\right|_{0} \simeq\left|\nabla \times\left(\mathbf{H}-\mathbf{H}_{h}\right)\right|_{0} .
$$

Thus, following the electric case, the quantity $\left|\nabla \times\left(\mathbf{H}-\mathbf{H}_{h}\right)\right|_{0}$ will be referred to as the "electric field" error, while we will denote $\left|\mathbf{H}-\mathbf{H}_{h}\right|_{0}$ as the "magnetic field" error.

Our analysis will be analogous to that of the electric field formulation, and we shall provide fewer details. In particular, the proofs of Theorems 12 and 13 as well as Corollaries 14 and 16 are omitted, because they are analogous to their E-formulation counterparts.

As for the electric field formulation, we require some regularity on the right-hand sides. Following Theorem 4, we assume that $\sigma^{-1} \mathbf{J} \in \mathbf{H}_{0}(\operatorname{curl}, \Omega)$ and $\mathbf{M} \in \mathbf{H}_{0}(\operatorname{div}, \Omega)$. We remark that the regularity assumptions are different from those of the electric field formulation. Though we do not investigate numerically this aspect in this work, it might be useful to select between the $\mathbf{E}$ - and $\mathbf{H}$-formulations depending on the type of electromagnetic sources in presence.

2.4.1. Interpolation error estimates. For $\mathbf{j}, \mathbf{m} \in \mathbf{L}^{2}(\Omega)$, let $\mathcal{H}(\mathbf{j}, \mathbf{m})$ be the unique element $\mathcal{H}(\mathbf{j}, \mathbf{m}) \in \mathbf{H}(\mathrm{curl}, \Omega)$ such that

$$
b_{\mathbf{H}}(\mathcal{H}(\mathbf{j}, \mathbf{m}), \mathbf{v})=(\mathbf{m}, \mathbf{v})+\left(\sigma^{-1} \mathbf{j}, \nabla \times \mathbf{v}\right) \quad \forall \mathbf{v} \in \mathbf{H}(\operatorname{curl}, \Omega) .
$$

We define

$$
\eta_{h}^{\mathbf{E}}=\sup _{\sigma^{-1} \mathbf{j} \in \mathbf{H}_{0}(\operatorname{curl}, \Omega), \mathbf{m} \in \mathbf{L}^{2}(\Omega)} \frac{\left|\nabla \times\left(\mathcal{H}(\mathbf{j}, \mathbf{m})-\Pi_{h} \mathcal{H}(\mathbf{j}, \mathbf{m})\right)\right|_{0}}{\left\|\sigma^{-1} \mathbf{j}\right\|_{\text {curl }}+|\mathbf{m}|_{0}}
$$

and

$$
\eta_{h}^{\mathbf{H}}=\sup _{\mathbf{j} \in \mathbf{L}^{2}(\Omega), \mathbf{m} \in \mathbf{H}_{0}(\operatorname{div}, \Omega)} \frac{\left|\mathcal{H}(\mathbf{j}, \mathbf{m})-\Pi_{h} \mathcal{H}(\mathbf{j}, \mathbf{m})\right|_{0}}{|\mathbf{j}|_{0}+\|\mathbf{m}\|_{\text {div }}}
$$

which characterize the approximation properties of the interpolant. Note that the conditions $\sigma^{-1} \mathbf{j} \in H(\operatorname{curl}, \Omega)$ and $\mathbf{m} \in H($ div,$\Omega)$ are included in the definitions of $\eta_{h}^{\mathbf{E}}$ and $\eta_{h}^{\mathbf{H}}$, respectively. 
THEOREM 12. It holds that

$$
\eta_{h}^{\mathbf{H}} \lesssim h
$$

for both $\mathscr{P}$-fitting and non- $\mathscr{P}$-fitting meshes.

If $\mathcal{T}_{h}$ is a $\mathscr{P}$-fitting mesh, we have

$$
\eta_{h}^{\mathbf{E}} \lesssim h^{s}
$$

for all $0<s<\tau(\sigma)$.

If $\tilde{\mathcal{T}}_{h}$ is not a $\mathscr{P}$-fitting mesh, then we only have

$$
\eta_{h}^{\mathbf{E}} \lesssim h^{t}
$$

for all $0<t<\tilde{\tau}(\sigma)$.

2.4.2. Electric field error estimates. In the following theorem, we apply Céa's lemma to prove the convergence of the FE solution.

Theorem 13. There exists a unique $\mathbf{H}_{h} \in \mathbf{V}_{h}$ satisfying (47). Furthermore, we have

$$
\left\|\mathbf{H}-\mathbf{H}_{h}\right\|_{\text {curl }} \lesssim\left(\eta_{h}^{\mathbf{H}}+\eta_{h}^{\mathbf{E}}\right)\left(\left\|\sigma^{-1} \mathbf{J}\right\|_{\text {curl }}+\|\mathbf{M}\|_{\text {div }}\right) .
$$

As in the electric field formulation, the above result is optimal in terms of the electric field approximation. We record this result in Corollary 14. We recall that, since $\mathbf{E}=\sigma^{-1}(\mathbf{J}-\nabla \times \mathbf{H}), \tilde{\mathbf{E}}_{h}=\sigma^{-1}\left(\mathbf{J}-\nabla \times \mathbf{H}_{h}\right)$ is a natural approximation of the electric field, and we have

$$
\left|\mathbf{E}-\tilde{\mathbf{E}}_{h}\right|_{0} \simeq\left|\nabla \times\left(\mathbf{H}-\mathbf{H}_{h}\right)\right|_{0} .
$$

Corollary 14. If $\mathcal{T}_{h}$ is a $\mathscr{P}$-fitting mesh, it holds that

$$
\left|\nabla \times\left(\mathbf{H}-\mathbf{H}_{h}\right)\right|_{0} \lesssim h^{s}\left(\left\|\sigma^{-1} \mathbf{J}\right\|_{\text {curl }}+\|\mathbf{M}\|_{\text {div }}\right)
$$

for all $0<s<\tau(\sigma)$.

If $\tilde{\mathcal{T}}_{h}$ is not $\mathscr{P}$-fitting, then we have

$$
\left|\nabla \times\left(\mathbf{H}-\mathbf{H}_{h}\right)\right|_{0} \lesssim h^{t}\left(\left\|\sigma^{-1} \mathbf{J}\right\|_{\text {curl }}+\|\mathbf{M}\|_{\text {div }}\right)
$$

for all $0<t<\tilde{\tau}(\sigma)$.

2.4.3. Magnetic field error estimates. As in the electric field formulation, our key result is an improved convergence rate for the magnetic field $L^{2}$ error.

THEOREM 15. It holds that

$$
\left|\mathbf{H}-\mathbf{H}_{h}\right|_{0} \lesssim\left(\eta_{h}^{\mathbf{H}}+\left(\eta_{h}^{\mathbf{E}}+\eta_{h}^{\mathbf{H}}+h\right)\left(\eta_{h}^{\mathbf{E}}+\eta_{h}^{\mathbf{H}}\right)\right)\left(\left\|\sigma^{-1} \mathbf{J}\right\|_{c u r l}+\|\mathbf{M}\|_{d i v}\right) .
$$

Proof. The proof is again based on a modified version of the Aubin-Nitsche duality argument. We first introduce the Hodge-Helmholtz decomposition of the error vector:

$$
\mathbf{H}-\mathbf{H}_{h}=\nabla p+\boldsymbol{\phi}
$$


with $p \in H^{1}(\Omega), \phi \in \mathbf{H}(\operatorname{curl}, \Omega), \nabla \cdot \phi=0$, and $\phi \cdot \mathbf{n}=0$. Then, we have

$$
\Pi_{h} \mathbf{H}-\mathbf{H}_{h}=\Pi_{h}\left(\mathbf{H}-\mathbf{H}_{h}\right)=\nabla r^{h}(p)+\Pi_{h} \phi .
$$

Hence it remains to bound $|\nabla p|_{0}$ and $|\phi|_{0}$. To this end, we introduce $\boldsymbol{\psi}=\overline{\mathcal{H}(\mathbf{j}, \mathbf{m})}$, with $\mathbf{j}=\mathbf{0}$ and $\mathbf{m}=\overline{\boldsymbol{\phi}}$ so that, by Galerkin orthogonality,

$$
|\phi|_{0}^{2}=\left(\mathbf{H}-\mathbf{H}_{h}, \boldsymbol{\phi}\right)=b_{\mathbf{H}}\left(\mathbf{H}-\mathbf{H}_{h}, \boldsymbol{\psi}\right)=b_{\mathbf{H}}\left(\mathbf{H}-\mathbf{H}_{h}, \boldsymbol{\psi}-\Pi_{h} \boldsymbol{\psi}\right) .
$$

Since $\mathbf{0}=\mathbf{j} \in \mathbf{H}_{0}(\mathrm{curl}, \Omega)$, and because $\nabla \cdot \boldsymbol{\phi}=0$ and $\boldsymbol{\phi} \cdot \mathbf{n}=0$, we have $\mathbf{m}=\bar{\phi} \in \mathbf{H}_{0}(\operatorname{div}, \Omega)$ and $\|\mathbf{m}\|_{\text {div }}=|\phi|_{0}$. As a result, we obtain

$$
\begin{aligned}
\left|b_{\mathbf{H}}\left(\mathbf{H}-\mathbf{H}_{h}, \boldsymbol{\psi}-\Pi_{h} \boldsymbol{\psi}\right)\right| & \lesssim \eta_{h}^{\mathbf{H}}\left|\mathbf{H}-\mathbf{H}_{h}\right|_{0}\left|\boldsymbol{\psi}-\Pi_{h} \boldsymbol{\psi}\right|_{0}+\eta_{h}^{\mathbf{E}}\left|\nabla \times\left(\mathbf{H}-\mathbf{H}_{h}\right)\right|_{0}\left|\nabla \times\left(\boldsymbol{\psi}-\Pi_{h} \boldsymbol{\psi}\right)\right|_{0}, \\
& \lesssim \eta_{h}^{\mathbf{H}}\left|\mathbf{H}-\mathbf{H}_{h}\right|_{0}|\boldsymbol{\phi}|_{0}+\eta_{h}^{\mathbf{E}}\left|\nabla \times\left(\mathbf{H}-\mathbf{H}_{h}\right)\right|_{0}|\boldsymbol{\phi}|_{0},
\end{aligned}
$$

so that

$$
|\phi|_{0} \lesssim \eta_{h}^{\mathbf{H}}\left|\mathbf{H}-\mathbf{H}_{h}\right|_{0}+\eta_{h}^{\mathbf{E}}\left|\nabla \times\left(\mathbf{H}-\mathbf{H}_{h}\right)\right|_{0} .
$$

Using Theorem 13, we obtain

$$
|\phi|_{0} \lesssim\left(\eta_{h}^{\mathbf{H}}+\eta_{h}^{\mathbf{E}}\right)^{2}\left(\left\|\sigma^{-1} \mathbf{J}\right\|_{\text {curl }}+\|\mathbf{M}\|_{\text {div }}\right) .
$$

For the estimate of $|\nabla p|_{0}$, as $(\phi, \nabla p)=0$, we can write

$$
\begin{aligned}
|\nabla p|_{0}^{2} & =\left(\mathbf{H}-\mathbf{H}_{h}, \nabla p\right) \\
& =\left(\Pi_{h} \mathbf{H}-\mathbf{H}_{h}, \nabla p\right)+\left(\mathbf{H}-\Pi_{h} \mathbf{H}, \nabla p\right) \\
& =\left(\nabla r^{h}(p)+\Pi_{h} \boldsymbol{\phi}, \nabla p\right)+\left(\mathbf{H}-\Pi_{h} \mathbf{H}, \nabla p\right) . \\
& =\left(\nabla r^{h}(p), \nabla p\right)+\left(\Pi_{h} \boldsymbol{\phi}-\boldsymbol{\phi}, \nabla p\right)+\left(\mathbf{H}-\Pi_{h} \mathbf{H}, \nabla p\right) .
\end{aligned}
$$

Now, we observe that, if $q^{h} \in S^{h}$, then $\nabla \times \nabla q^{h}=\mathbf{0}$, and we have

$$
\left(\nabla p, \nabla q^{h}\right)=\left(\mathbf{H}-\mathbf{H}_{h}, \nabla q^{h}\right)=b_{\mathbf{H}}\left(\mathbf{H}-\mathbf{H}_{h}, \nabla q^{h}\right)=0 .
$$

In particular, $\left(\nabla r^{h}(p), \nabla p\right)=0$, and we have

$$
|\nabla p|_{0}^{2}=\left(\Pi_{h} \boldsymbol{\phi}-\boldsymbol{\phi}, \nabla p\right)+\left(\mathbf{H}-\Pi_{h} \mathbf{H}, \nabla p\right),
$$

so that

$$
|\nabla p|_{0} \leq\left|\boldsymbol{\phi}-\Pi_{h} \phi\right|_{0}+\left|\mathbf{H}-\Pi_{h} \mathbf{H}\right|_{0} .
$$

Since $\nabla \cdot \phi=0$ and $\phi \cdot \mathbf{n}=0$, we have (see [22, Theorem I.3.9]) $\phi \in \mathbf{H}^{1}(\Omega)$ and $|\phi|_{1} \lesssim|\nabla \times \phi|_{0}$. Then,

$$
\left|\phi-\Pi_{h} \phi\right|_{0} \lesssim h|\phi|_{1} \lesssim h|\nabla \times \phi|_{0}=h\left|\nabla \times\left(\mathbf{H}-\mathbf{H}_{h}\right)\right|_{0} .
$$

As a result, we obtain

$$
|\nabla p|_{0} \lesssim h\left|\nabla \times\left(\mathbf{H}-\mathbf{H}_{h}\right)\right|_{0}+\left|\mathbf{H}-\Pi_{h} \mathbf{H}\right|_{0} \lesssim h\left(\eta_{h}^{\mathbf{E}}+\eta_{h}^{\mathbf{H}}\right)+\eta_{h}^{\mathbf{H}} .
$$

Error estimate (54) then follows from (55) and (56). 
As in the electric field formulation, Corollary 16 provides optimal convergence rates in the general case.

COROLlary 16. Assume that $\tau(\sigma) \leq 1 / 2$. Then it holds that

$$
\left|\mathbf{H}-\mathbf{H}_{h}\right|_{0} \lesssim h^{2 s}\left(\left\|\sigma^{-1} \mathbf{J}\right\|_{\text {curl }}+\|\mathbf{M}\|_{\text {div }}\right)
$$

for all $0<s<\tau(\sigma)$ for both fitting and nonfitting meshes.

If $\tau(\sigma)>1 / 2$, then we have

$$
\left|\mathbf{H}-\mathbf{H}_{h}\right|_{0} \lesssim h\left(\left\|\sigma^{-1} \mathbf{J}\right\|_{\text {curl }}+\|\mathbf{M}\|_{\text {div }}\right)
$$

for fitting meshes and

$$
\left|\mathbf{H}-\mathbf{H}_{h}\right|_{0} \lesssim h^{1-\delta}\left(\left\|\sigma^{-1} \mathbf{J}\right\|_{c u r l}+\|\mathbf{M}\|_{\text {div }}\right)
$$

for nonfitting meshes.

\section{Numerical experiments.}

3.1. Settings. In this section, we describe the $2 \mathrm{D}$ numerical experiments we have selected to illustrate our error estimates proved in the previous section. For the sake of simplicity, we consider as computational domain the unit square $\Omega=(0,1)^{2}$, and denote by $(x, z)$ the coordinate system.

The target applications for the proposed method are borehole logging simulations. For this type of application, the domain is the whole space, but it is artificially bounded for numerical computations. Since sources are localized, the electromagnetic fields exhibit an exponential decay as a function of the distance from the source, and truncating the domain only introduces a small modelization error. The choice of the domain size is guided by the so-called skin-depth relation, which links the frequency, the conductivity, and the decay rate of the electromagnetic fields. We refer the reader to $[44]$.

The value of the conductivity ranges from $10^{-3} \mathrm{Sm}^{-1}$ to $5 \mathrm{Sm}^{-1}$ in our numerical experiments. Hence, based on the skin-depth relation, we select the frequency $f=2$ Mhz (and $\omega=2 \pi f$ ). In this way, the frequency, the domain size, and the conductivity model are representative of borehole logging applications.

Fitting and nonfitting meshes are considered in the following numerical experiments. When a nonfitting mesh is used, the "exact quadrature method" $[11,13]$ is used to integrate the coefficients of the FE linear system exactly.

3.1.1. Meshes and finite element spaces. We use Cartesian grid based meshes with square cells. Hence, given $h=1 / n$, the mesh $\mathcal{T}_{h}$ is defined as

$$
\mathcal{T}_{h}=\{K=((i-1) h, i h) \times((j-1) h, j h), \quad 1 \leq i, j \leq n\} .
$$

Because meshes $\mathcal{T}_{h}$ are made of squares, we need to slightly modify the definitions of the Nédélec's element spaces given in section 2. Hence, we introduce

$$
\mathbf{V}_{h}=\left\{\mathbf{v}_{h} \in \mathbf{H}(\operatorname{curl}, \Omega) \quad\left|\quad \mathbf{v}_{h}\right|_{K} \in Q_{0} \times Q_{1}+Q_{1} \times Q_{0} \quad \forall K \in \mathcal{T}_{h}\right\},
$$

where $Q_{0}=\operatorname{span}\{1\}$ and $Q_{1}=\operatorname{span}\{1, x, z, x z\}$. We also define $\mathbf{V}_{h, 0}=\mathbf{V}_{h} \cap$ $\mathbf{H}_{0}(\operatorname{curl}, \Omega)$. 


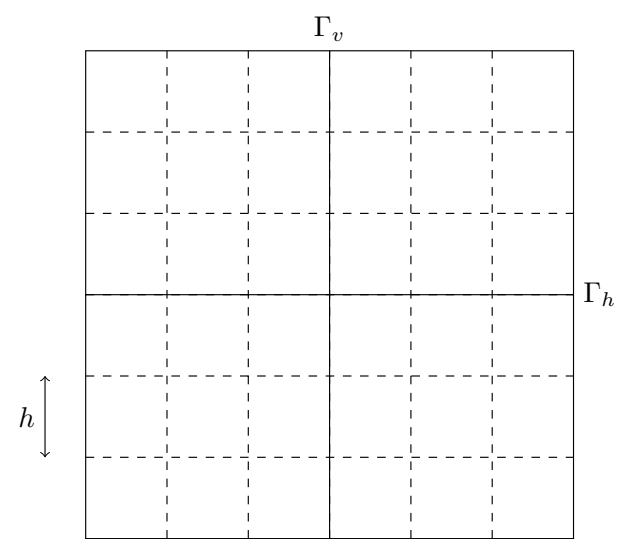

(a) Fitting mesh $(n=6)$

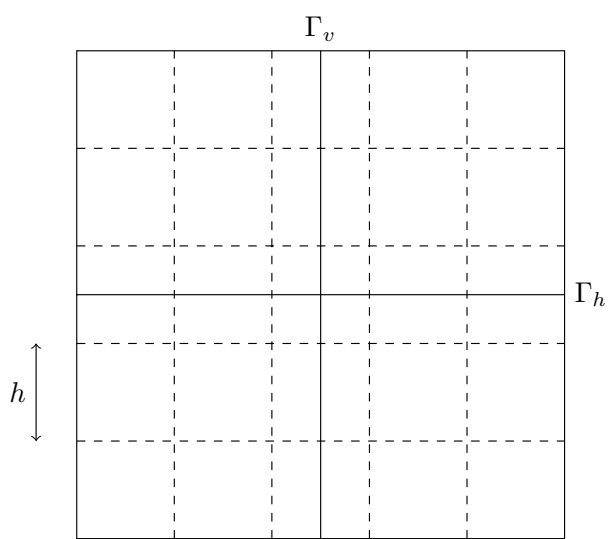

(b) Nonfitting mesh $(n=5)$

FIG. 3. Fitting and nonfitting meshes.

3.1.2. Error computations. In order to compute numerical errors, we will use an FE solution computed on a fine mesh. In our convergence curves, the values of $n$ range from 512 to 2048 . We select $n=4096$ to compute the reference FE solution.

Also, an important part of our analysis relies on the comparison between fitting and nonfitting meshes. In order to carry out such a comparison, we select a piecewise constant conductivity $\sigma$ that is discontinuous only at the interfaces $\Gamma_{h}=(0,1) \times\{0.5\}$ and $\Gamma_{v}=\{0.5\} \times(0,1)$. Then, it is easily seen that if $n$ is an even integer, both $\Gamma_{h}$ and $\Gamma_{v}$ are covered by mesh edges, so that the mesh is fitting. On the other hand, if $n$ is an odd integer, the two interfaces lie in the interior of some mesh cells, leading to a nonfitting mesh. Thus, when comparing fitting and nonfitting meshes, we will compare meshes with an even number of subdivisions $n$ with meshes having an odd number of subdivisions. We refer the reader to Figure 3, where the difference between fitting and nonfitting meshes is highlighted.

3.2. Validation experiments. We first present two validation experiments. For both experiments, the conductivity parameter is given by

$$
\sigma(x, z)= \begin{cases}1 & \text { if } x<0.5, z>0.5 \\ \sigma_{b} & \text { if } x>0.5, z>0.5 \\ \sigma_{b} & \text { if } x<0.5, z<0.5 \\ 1 & \text { if } x>0.5, z<0.5\end{cases}
$$

where $\sigma_{b}$ is a chosen constant, and we use the right-hand side $\mathbf{J}=(1,0,1)$.

Since we are in a 2D setting, singularities do not occur in the TE-polarization; hence we focus on the TM-polarization. For this polarization, we have $\mathbf{E}=\left(E_{x}, 0, E_{z}\right)$ and $\mathbf{H}=\left(0, H_{y}, 0\right)$. Furthermore an analytical expression of $\tau(\sigma)$ is available from [17]. Then, we discretize the problem using formulation (32) for $\left(E_{x}, E_{z}\right)$ and compute an approximation of $H_{y}$ by postprocessing (compare with (33))

$$
H_{y, h}=\mathbf{H}_{h}=\frac{1}{i \omega \mu_{0}} \operatorname{curl}\left(E_{x, h}, E_{z, h}\right),
$$

where $\left(E_{x, h}, E_{z, h}\right)$ is the unique solution of (32).

For this choice of conductivity parameter, we have an analytical expression for the regularity coefficient of the electric field. Based on Theorem 8.1 of [17], the expected 
regularity of the solution is $\mathbf{E} \in \mathbf{H}^{s}(\Omega)$ for $0 \leq s<\tau(\sigma)$, where

$$
\tau(\sigma)=\frac{4}{\pi} \arctan \left(\sqrt{\sigma_{b}}\right) .
$$

Here, we measure the global $L^{2}$ error norm, i.e.,

$$
e_{\Omega}(\phi)=\frac{\left|\phi-\phi_{h}\right|_{0}}{|\phi|_{0}}, \quad \phi=\mathbf{E}, H_{y} .
$$

3.2.1. Experiment A. We first consider the case where $\tau(\sigma)>1 / 2$. Hence, we select the value $\sigma_{b}=0.5$, so that $(60)$ yields $\tau(\sigma) \simeq 0.78$.

As seen in Figure 4, we observe a convergence rate in $\mathcal{O}\left(h^{\tau(\sigma)}\right)$ for the electric field approximation when using fitting meshes.

We also observe in Figure 4 that when nonfitting meshes are used, the electric field approximation converges only as $\mathcal{O}\left(h^{1 / 2}\right)$. This is in accordance with our analysis. Indeed, since $\tau(\sigma)>1 / 2$, we have $\tilde{\tau}(\sigma)=1 / 2$.

Finally, Figure 4 shows that the convergence for the magnetic field is in $\mathcal{O}(h)$ for both fitting and nonfitting meshes, as expected.

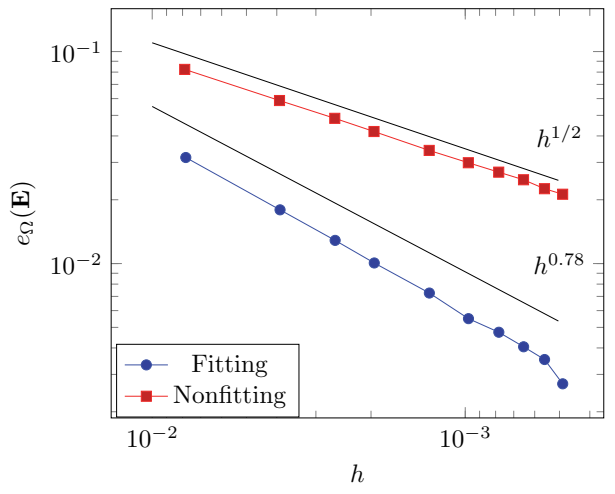

(a) Electric field errors

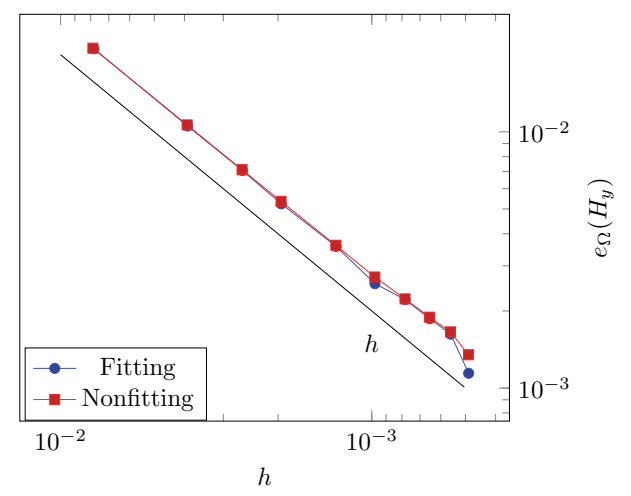

(b) Magnetic field errors

FIG. 4. Experiment A.

3.2.2. Experiment B. We now consider the case $\tau(\sigma)<1 / 2$. We set $\sigma_{b}=0.1$, so that $\tau(\sigma) \simeq 0.39$.

As Figure 5 shows, nonfitting meshes exhibit the expected results exactly. Indeed, the convergence rate for the electric field is $\tilde{\tau}(\sigma)=\tau(\sigma) \simeq 0.39$, and the magnetic field converges as $2 \tilde{\tau}(\sigma)$.

For fitting meshes, as shown in Figure 5, both the electric and magnetic approximations converge faster than expected, at rates $1 / 2$ and 1 , respectively.

These results are rather surprising since we observe that the electric field approximation converges faster than $\tau(\sigma)$, which is the convergence rate of the best approximation. This is not in contradiction with our theoretical analysis, since we only show upper bounds. However, these results question the optimality of the presented analysis.

The authors believe, however, that the proposed error estimates are optimal, and that the observed superconvergence rate can be explained by a preasymptotic effect. 
Indeed, we can decompose $\mathbf{E}$ as

$$
\mathbf{E}=\mathbf{E}_{s}+\mathbf{E}_{r},
$$

where $\mathbf{E}_{s} \in \mathbf{H}^{\tau(\sigma)}(\Omega)$ and $\mathbf{E}_{r} \in \mathbf{H}^{1}(\Omega)$ correspond to singular and regular parts of the electric field. Consequently, if $\|\mathbf{E}\|_{s, \Omega} \lll\left\|\mathbf{E}_{r}\right\|_{1, \Omega}$, it is reasonable to expect a superconvergence phenomena as far as the mesh sizes $h$ are relatively coarse.

This superconvergence result is specific to this particular right-hand side. We show in subsection 3.3.3 a test with another right-hand side in which the predicted behavior is observed for both fitting and nonfitting meshes.

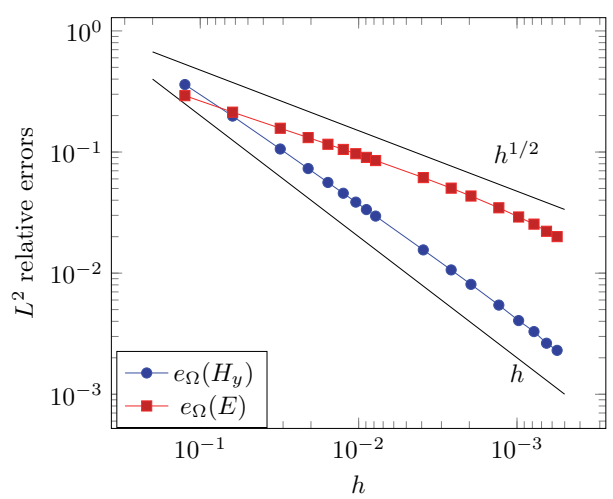

(a) Fitting meshes

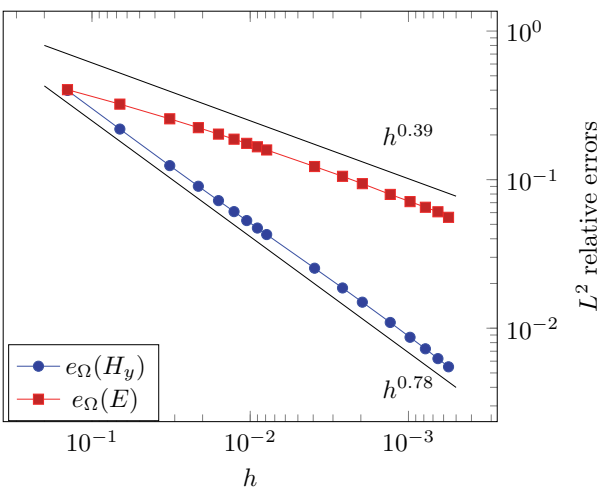

(b) Nonfitting meshes

FIG. 5. Experiment B.

3.3. "Geophysical" experiments. We provide another set of numerical experiments directed toward geophysical applications. The sources are Gaussian loads representing electric transmitters. The $L^{2}$ error is measured locally in a small area representing a magnetic or electric receiver.

3.3.1. Experiment 1. We start with a test case using the TE-polarization. We consider a conductivity model featuring two layers, separated by a planar interface. In the top layer, the conductivity takes the value $1 \mathrm{Sm}^{-1}$. In the bottom layer, we consider three different tests with different values $\sigma_{b} \in\{0.9,0.1,0.001\} \mathrm{Sm}^{-1}$ of $\sigma$. In that way, we are able to analyze the influence of the conductivity contrast.

The interface is located at depth $z=0.5 \mathrm{~m}$. Hence, the conductivity is defined as

$$
\sigma(x, z)=\left\{\begin{array}{lll}
1 & \mathrm{Sm}^{-1} & \text { if } z>0.5 \\
\sigma_{b} & \mathrm{Sm}^{-1} & \text { if } z<0.5
\end{array}\right.
$$

We consider the source $\mathbf{J}=(0, g, 0)$, where $g$ is a Gaussian load centered at $(0.4,0.4)$ :

$$
g(x, z)=\exp \left(-\frac{(x-0.4)^{2}+(z-0.4)^{2}}{\left(10^{-2}\right)^{2}}\right) .
$$

The electric field has only one nonzero component $\left(\mathbf{E}=\left(0, E_{y}, 0\right)\right)$, and the magnetic field takes the form $\mathbf{H}=\left(H_{x}, 0, H_{z}\right)$. Because the electric field has only one nonzero component, the electric field formulation employs Lagrange $H^{1}$-conforming elements. Since in this work we are mostly interested in Nédélec's elements, we will 
therefore focus on the magnetic field formulation. In other words, we approximate $\left(H_{x}, H_{z}\right)$ by using $(47)$ and compute a discrete electric field approximation as $E_{y, h}=$ $\sigma^{-1}\left(g-\operatorname{curl}\left(H_{x, h}, H_{z, h}\right)\right)$.

We measure the $L^{2}$ error in a subdomain $R=(0.6,0.8) \times(0.6,0.8)$ located "far" from the source center, in the bottom layer. Hence, we will measure the approximation errors as

$$
e_{R}(\phi)=\frac{\left|\phi-\phi_{h}\right|_{0, R}}{|\phi|_{0, R}}, \quad \phi=E_{y}, H_{x}, H_{z} .
$$

Physically, the quantity $\left.\phi\right|_{R}\left(\phi=E_{y}, H_{x}, H_{z}\right)$ can be understood as the measure of electromagnetic fields at a receiver location. Thus, the quantities $e_{R}(\phi)$ are actually a good indicator of the error on the quantity of interest in borehole applications.
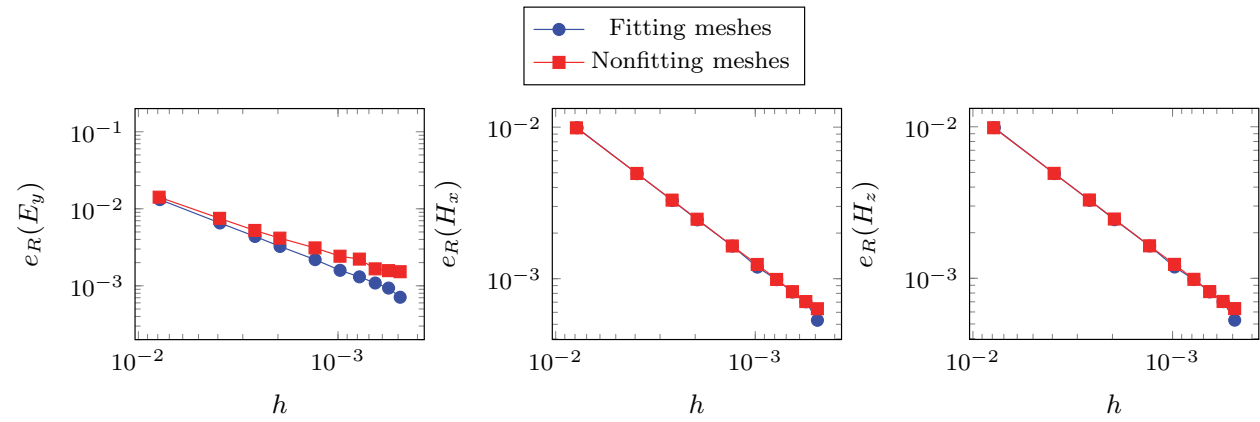

(a) $\sigma_{b}=0.9$
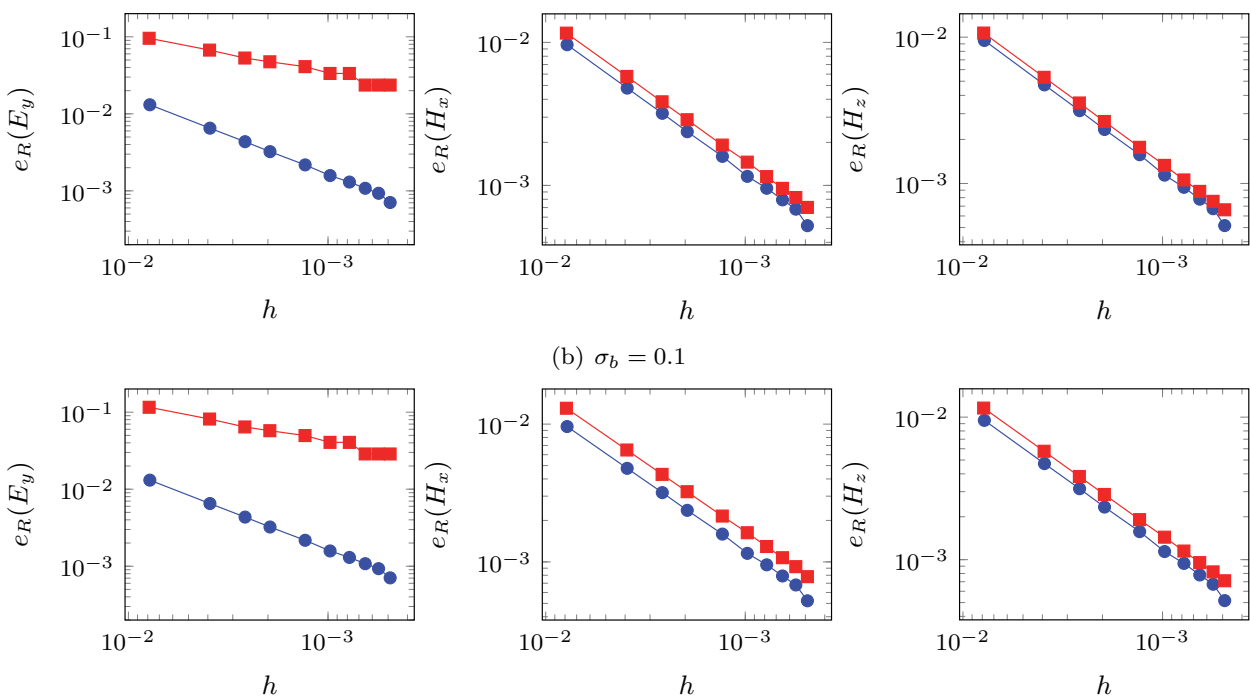

(c) $\sigma_{b}=0.001$

FIG. 6. Experiment 1.

Figure 6 shows the results of Experiment 1. The predicted convergence rates are observed. Indeed, we have $e_{R}\left(H_{x}\right) \lesssim h, e_{R}\left(H_{y}\right) \lesssim h$, and $e_{R}\left(E_{y}\right) \lesssim h^{1 / 2}$.

The case of a "small" contrast is represented by $\sigma_{b}=0.9$ in Figure 6(a). In this case, we observe similar results for fitting and nonfitting meshes, although some discrepancies are observed for the $e_{R}\left(E_{y}\right)$ case. Furthermore, we see that for nonfitting 
meshes, the convergence of the electric field is quasi-linear in some preasymptotic range and decreases asymptotically for small $h$.

In Figures 6(b) and (c), higher contrasts are considered. In these cases, the convergence rate of the electric field clearly decreases for nonfitting meshes, and we observe the predicted convergence rate $\mathcal{O}\left(h^{1 / 2}\right)$. For the magnetic field, the predicted convergence rate is respected, as the convergence is linear, both for fitting and nonfitting meshes. We see that the magnetic field error is greater by a constant factor for nonfitting meshes. Nonetheless, this factor is rather small and increases reasonably slowly with the conductivity contrast. Indeed, even for the highest contrast (1 to $\sigma_{b}=10^{-3}$ ) the accuracy loss due to the use of nonfitting meshes is limited to a factor of two.

3.3.2. Experiment 2. We now consider an experiment in the TM-polarization. We keep the same settings as in Experiment 1, except that the source now reads

$$
\mathbf{J}=(0,0, g)
$$

with the same Gaussian $g$ as before. The magnetic field has only one nonzero component $H_{y}$, and we have $\mathbf{E}=\left(E_{x}, 0, E_{z}\right)$. We will therefore focus on the electric field formulation (32).

Results are presented in Figure 7. The conclusions are similar to those obtained for the case of TE-polarization presented in Experiment 1. First, we observe that the convergence rates predicted by the theoretical analysis are strictly respected. The magnetic field approximation convergence is linear for fitting and nonfitting meshes, and the electric field convergence rate is at its worst $1 / 2$.

Again, we see that for a small conductivity contrast, the electric field approximation with nonfitting meshes has quasi-linear convergence in a preasymptotic range and deteriorates as $h$ goes to zero. Also, concerning the magnetic field, the nonfitting mesh error is higher than the fitting mesh error by a constant factor that grows with the conductivity contrast. Nevertheless, this constant remains small, even for the highest considered contrast.

An additional observation is that even for nonfitting meshes, the convergence of $e_{R}\left(E_{x}\right)$ is linear. Indeed, the nonfitting mesh error is higher than the fitting mesh error only by a constant factor. Such a constant increases with the conductivity contrast. While this enhanced accuracy is not proved in our theoretical study, it is not completely surprising, since $E_{x}$ is the component of the electric field tangential to the interface, and it is therefore continuous across the interface.

3.3.3. Experiment 3. In this experiment, we consider a conductivity model with crossing interfaces, generating a singular solution; see subsection 3.2. It is defined by

$$
\sigma(x, z)= \begin{cases}1 & \text { if } x<0.5, z>0.5 \\ 0.001 & \text { if } x>0.5, z>0.5 \\ 0.1 & \text { if } x<0.5, z<0.5 \\ 5 & \text { if } x>0.5, z<0.5\end{cases}
$$

As before, since we are in a 2D setting, singularities do not occur in the TEpolarization; for this reason, we only consider the TM-polarization and discretize the problem using formulation (32). We keep the same source used in Experiment 2.

As in subsection 3.2, the singularity takes place at the point where the two interfaces cross, namely at point $(0.5,0.5)$. In order to take into account as much as 

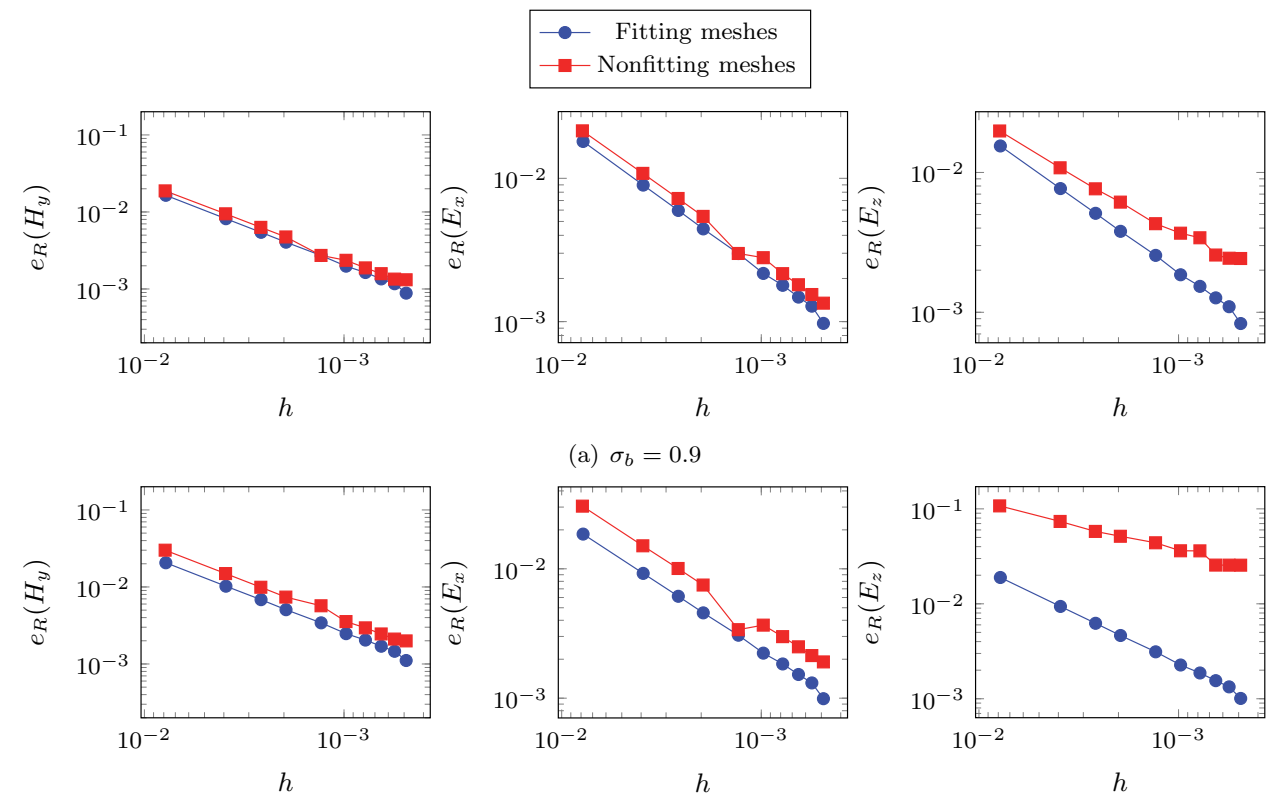

(a) $\sigma_{b}=0.9$
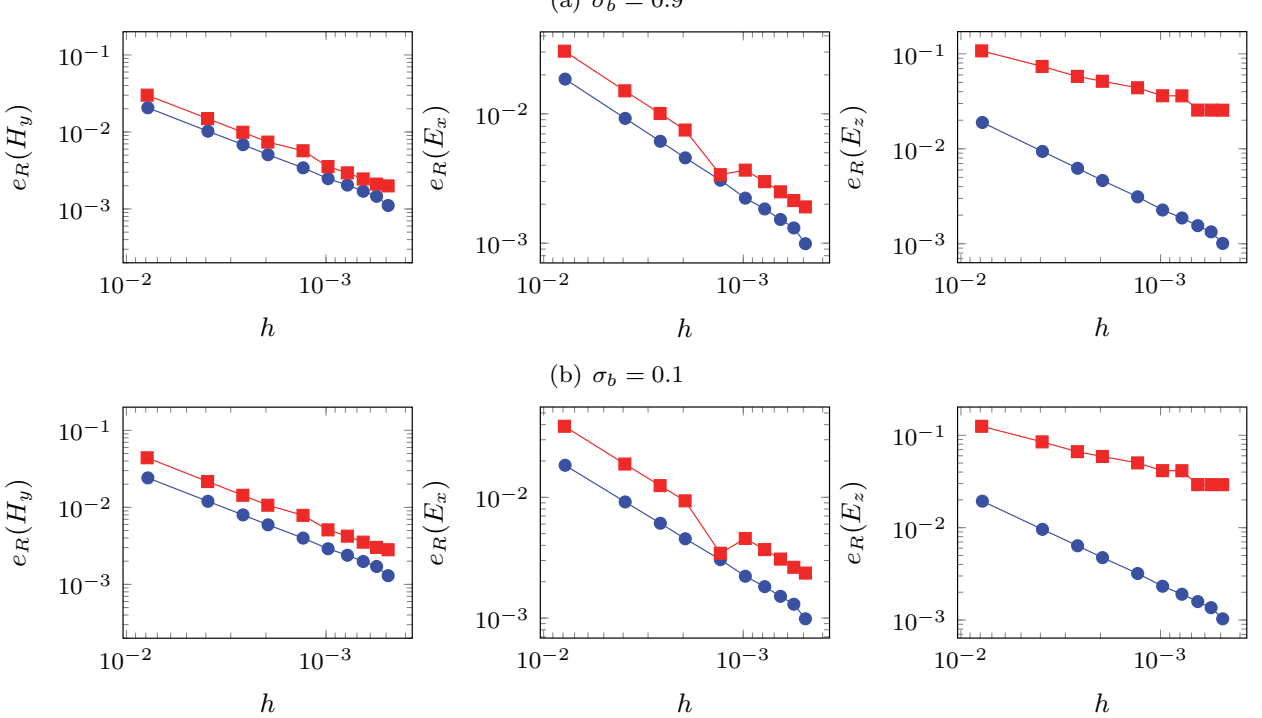

(b) $\sigma_{b}=0.1$

(c) $\sigma_{b}=0.001$

FIG. 7. Experiment 2.

possible the effect of the singularity in our error measurements, we measure the relative $L^{2}$ errors on the set $\tilde{R}=(0.5,0.7) \times(0.5,0.7)$. This choice can be physically interpreted as a receiver located close to the singular point.

Results are presented in Figure 8. We observe that the convergence rates predicted by the error analysis are respected. The magnetic field error decreases as $\mathcal{O}\left(h^{2 s}\right)$ with $s=1 / 3$, while the electric field error only behaves as $\mathcal{O}\left(h^{s}\right)$. These observations are valid for both fitting and nonfitting meshes. In particular, this experiment supports our claim that convergence rates are the same for fitting and nonfitting meshes in the presence of a strong singularity.

The accuracy obtained with fitting and nonfitting meshes is equivalent for the electric field. For the magnetic field, the accuracy loss due to the use of nonfitting meshes is a constant factor. In this test case, fitting meshes are about twice as accurate as nonfitting meshes for the approximation of the magnetic field.

Conclusion. In this work, we developed a convergence theory for the general 3D Maxwell's system with constant permeability and permittivity and a piecewise constant conductivity. The theory is illustrated with 2D numerical experiments. All 


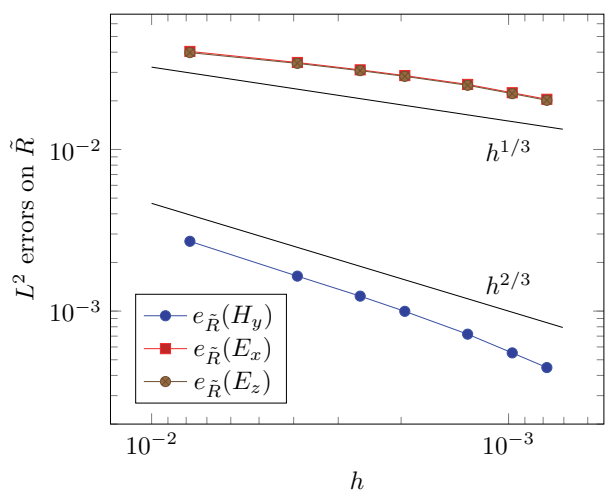

(a) Fitting meshes

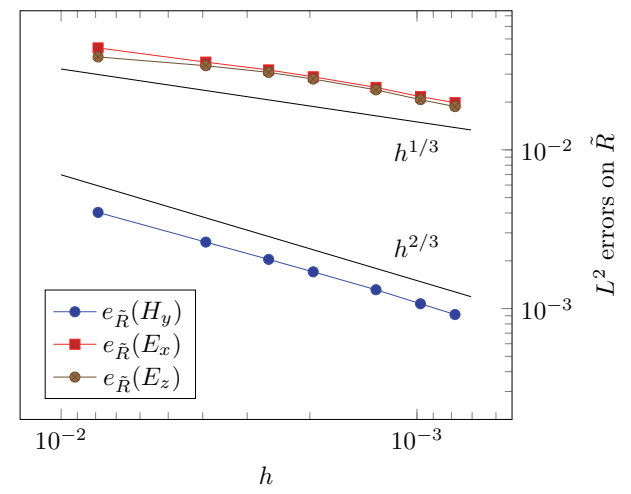

(b) Nonfitting meshes

FIG. 8. Experiment 3.

parameters are scalar valued. We have focused on proving error estimates with optimal convergence rates for first order Nédélec's elements. The main novelty of our analysis is that the convergence rates derived for the electric and magnetic fields are different. If the electric field has regularity $H^{s}$, it is well known that the electric field approximation converges as $\mathcal{O}\left(h^{s}\right)$. Our key result is an improved error estimate for the magnetic field, where we show that the approximation converges as $\mathcal{O}\left(h^{\min (1,2 s)}\right)$. As detailed in section 2, this result holds for both the E- and $\mathrm{H}$-formulations of Maxwell's equations.

We have observed in section 3 that, in the particular case of a layered medium, our general result implies that the convergence rate for the magnetic field is always linear, regardless of the use of fitting or nonfitting meshes.

We have illustrated the main features of our convergence analysis using 2D examples, both in TE- and TM-polarizations. The predicted convergence rates are observed in all numerical experiments, which makes us confident that our results are optimal. We have also carefully compared fitting and nonfitting meshes. As predicted by the convergence analysis, the results obtained for the magnetic field are similar for both types of meshes. Thus, we advocate the use of nonfitting meshes to approximate the magnetic field without a significant loss in accuracy.

In layered media, we have additionally observed that when the conductivity contrast is small, the electric field convergence rate is linear in some preasymptotic range. Also, we have seen that the convergence rate of the component of the electric field tangential to the interface is linear. These two features are not included in our present analysis, and they should be the object of future investigations focused on layered media only.

Although we have considered the 3D Maxwell's system for the proofs, the ideas are rather general and should apply to other systems, such as 2.5D and 2D Maxwell's systems, with minor modifications. Also, the "spirit" of the proof showing that the magnetic field approximation is more accurate might apply to other discretization strategies, such as finite difference schemes and/or numerical methods including averaging techniques.

Future work will be guided towards numerical experimentations on $2.5 \mathrm{D}$ and $3 \mathrm{D}$ Maxwell's equations in more realistic settings. 


\section{REFERENCES}

[1] R. A. Adams and J. J. Fournier, Sobolev Spaces, 2nd ed., Academic Press, 2003.

[2] A. Abubakar, T. Habashy, V. Druskin, L. Knizhnerman, and D. Alumbaugh, 2.5 D forward and inverse modeling for interpreting low-frequency electromagnetic measurements, Geophysics, 73 (2008), pp. F165-F177.

[3] D. B. AvdeEv, Three-dimensional electromagnetic modelling and inversion from theory to application, Surv. Geophys., 26 (2005), pp. 767-799.

[4] I. BabušKa, G. CAloz, And J. E. Osborn, Special finite element methods for a class of second order elliptic problems with rough coefficients, SIAM J. Numer. Anal., 31 (1994), pp. 945-981, https://doi.org/10.1137/0731051.

[5] G. E. Backus, Long-wave anisotropy produced by horizontal layering, J. Geophys. Res., 67 (1962), pp. 4427-4440.

[6] H. Barucq, T. Chaumont-Frelet, and C. Gout, Stability analysis of heterogeneous Helmholtz problems and finite element solution based on propagation media approximation, Math. Comp., 86 (2016), pp. 2129-2157.

[7] A. Bonito, J. L. Guermond, and F. Luddens, Regularity of the Maxwell equations in heterogeneous media and Lipschitz domains, J. Math. Anal. Appl., 408 (2013), pp. 498-512.

[8] M. Bourlard, M. Dauge, M.-S. Lubuma, and S. Nicaise, Coefficients of the singularities for elliptic boundary value problems on domain with conical points. III: Finite element methods on polygonal domains, SIAM J. Numer. Anal., 29 (1992), pp. 136-155, https:// doi.org/10.1137/0729009.

[9] S. C. Brenner, F. LI, AND L. Y. Sung, A locally divergence-free nonconforming finite element method for the time-harmonic Maxwell equations, Math. Comp., 76 (2006), pp. 573-595.

[10] Y. Capdeville, L. Guillot, and J.-J. Marigo, 2-D non-periodic homogenization to upscale elastic media for P-SV waves, Geophys. J. Int., 182 (2010), pp. 903-922.

[11] T. Chaumont-Frelet, Finite Element Approximation of Helmholtz Problems with Application to Seimsic Wave Propagation, Ph.D. thesis, INSA Rouen and Inria EPI MAGIQUE3D, 2015.

[12] T. Chaumont-Frelet, On high order methods for the heterogeneous Helmholtz equation, Comput. Math. Appl., 72 (2016), pp. 2203-2225.

[13] T. Chaumont-Frelet, H. Barucq, H. Calandra, and C. Gout, A Multiscale Medium Approximation Method for the Propagation P-Waves in Highly Heterogeneous Geophysical Media, preprint HAL-01706454, 2018.

[14] P. G. Ciarlet, The Finite Element Method for Elliptic Problems, Classics Appl. Math. 40, SIAM, 2002, https://doi.org/10.1137/1.9780898719208.

[15] P. Ciarlet, S. Fliss, And C. Stohrer, On the approximation of electromagnetic fields by edge finite elements. Part 2: A heterogeneous multiscale method for Maxwell's equations, Comput. Math. Appl., 73 (2017), pp. 1900-1919.

[16] S. Constable and L. J. SRnka, An introduction to marine controlled-source electromagnetic methods for hydrocarbon exploration, Geophysics, 72 (2007), pp. WA3-WA12.

[17] M. Costabel, M. Dauge, And S. Nicaise, Singularities of Maxwell interface problems, M2AN Math. Model. Numer. Anal., 33 (1999), pp. 627-649.

[18] D. V. Ellis And J. M. Singer, Well Logging for Earth Scientists, Springer Science+Business Media, 2007.

[19] E. Engström And D. SJöBerg, A Comparison of Two Numerical Methods for Homogenization of Maxwell's Equations, Tech. report TEAT-7121, LUTEDX, 2004.

[20] A. ERn AND J. L. Guermond, Finite element quasi-interpolation and best approximation, M2AN Math. Model. Numer. Anal., 51 (2017), pp. 1367-1385.

[21] A. Ern and J. L. Guermond, Analysis of the edge finite element approximation of the Maxwell equations with low regularity solutions, Comput. Math. Appl., 75 (2018), pp. 918-932.

[22] V. Girault and P. A. Raviart, Finite Element Methods for Navier-Stokes Equations: Theory and Algorithms, Springer-Verlag, 1986.

[23] T. M. Habashy AND A. ABUBAKaR, A general framework for constraint minimization for the inversion of electromagnetic measurements, Prog. Electromag. Res., 46 (2004), pp. 265312 .

[24] T. M. Habashy and A. Abubakar, A generalized material averaging formulation for modelling of the electromagnetic fields, J. Electromag. Waves Appl., 21 (2007), pp. 1145-1159.

[25] P. Henning, M. Ohlberger, And B. Verfürth, A new heterogeneous multiscale method for time-harmonic Maxwell's equations, SIAM J. Numer. Anal., 54 (2016), pp. 3493-3522, https://doi.org/10.1137/15M1039225. 
[26] T. Y. Hou And X.-H. Wu, A multiscale finite element method for elliptic problems in composite materials and porous media, J. Comput. Phys., 134 (1997), pp. 169-189.

[27] F. Ihlenburg And I. BABUŠKa, Finite element solution of the Helmholtz equation with high wave number part I: The h-version of the FEM, Comput. Math. Appl., 30 (1995), pp. 9-37.

[28] P. Ciarlet, JR., On the approximation of electromagnetic fields by edge finite elements. Part 1: Sharp interpolation results for low-regularity fields, Comput. Math. Appl., 71 (2016), pp. 85-104.

[29] P. CiARlet, JR. AND J. Zou, Fully discrete finite element approaches for time-dependent Maxwell's equations, Numer. Math., 82 (1999), pp. 193-219.

[30] J. B. KelLer, A theorem on the conductivity of a composite medium, J. Math. Phys., 5 (1964), pp. $548-549$.

[31] J. Li, J. M. Melenk, B. Wohlmuth, And J. Zou, Optimal a priori estimates for higher order finite elements for elliptic interface problems, Appl. Numer. Math., 60 (2010), pp. 19-37.

[32] J. L. Lions and E. Magenes, Non-Homogeneous Boundary Value Problems and Applications, Springer, 1972.

[33] P. Monk, A finite element method for approximating the time-harmonic Maxwell equations, Numer. Math., 63 (1992), pp. 243-261.

[34] P. Monk, Finite Element Methods for Maxwell's Equations, Oxford University Press, 2003.

[35] S. Moskow, V. Druskin, T. Habashy, P. Lee, and S. Davydycheva, A finite difference scheme for elliptic equations with rough coefficients using a Cartesian grid nonconforming to interfaces, SIAM J. Numer. Anal., 36 (1999), pp. 442-464, https://doi.org/10.1137/ S0036142997318541.

[36] J. NÉdÉLEC, Mixed finite elements in $R^{3}$, Numer. Math., 35 (1980), pp. 315-341.

[37] N. C. Nguyen, J. Peraire, and B. Cockburn, Hybridizable discontinuous Galerkin methods for the time-harmonic Maxwell's equations, J. Comput. Phys., 230 (2011), pp. 7151-7175.

[38] S. NicAISE, Edge elements on anisotropic meshes and approximation of the Maxwell equations, SIAM J. Numer. Anal., 39 (2001), pp. 784-816, https://doi.org/10.1137/ S003614290036988X.

[39] D. Pardo, L. Demkowicz, C. Torres-Verdín, and M. Paszynski, Two-dimensional highaccuracy simulation of resistivity logging-while-drilling (LWD) measurements using a selfadaptive goal-oriented hp finite element method, SIAM J. Appl. Math., 66 (2006), pp. 20852106, https://doi.org/10.1137/050631732.

[40] D. Pardo, M. Paszynski, C. Torres-Verdín, and L. Demkowicz, Simulation of $3 D$ DC borehole resistivity measurements with a goal-oriented $h p$ finite-element method. Part I: Laterolog and LWD, J. Serbian Soc. Comput. Mech., 1 (2007), pp. 62-73.

[41] D. Pardo, C. Torres-Verdín, and L. Demkowicz, Feasibility study for $2 D$ frequencydependent electromagnetic sensing through casing, Geophysics, 72 (2007), pp. F111-F118.

[42] G. SANGalli, Capturing small scales in elliptic problems using a residual-free bubbles finite element method, Multiscale Model. Simul., 1 (2003), pp. 485-503, https://doi.org/10.1137/ S1540345902411402.

[43] J. SCHÖBERL, Commuting Quasi-Interpolation Operators for Mixed Finite Elements, Tech. report ISC-01-10-MATH, Texas A\&M University, 2001.

[44] F. Simpson and K. Bahr, Practical Magnetotellurics, Cambridge University Press, 2005.

[45] M. J. Wilt, D. L. Alumbaught, H. F. Morrison, A. Becker, K. H. Lee, and M. DeszczPAN, Crosswell electromagnetic tomography: System design considerations and field results, Geophysics, 60 (1995), pp. 871-885. 